\title{
Classification and Evolution of Bifurcation Curves for a Dirichlet-Neumann Boundary Value Problem and its Application
}

\author{
Da-Chang Kuo, Shin-Hwa Wang* and Yu-Hao Liang \\ In memory of Professor Hwai-Chiuan Wang
}

\begin{abstract}
We study the classification and evolution of bifurcation curves of positive solutions for the one-dimensional Dirichlet-Neumann boundary value problem

$$
\left\{\begin{array}{l}
u^{\prime \prime}(x)+\lambda f(u)=0, \quad 0<x<1, \\
u(0)=0, \quad u^{\prime}(1)=-c<0
\end{array}\right.
$$

where $\lambda>0$ is a bifurcation parameter and $c>0$ is an evolution parameter. We mainly prove that, under some suitable assumptions on $f$, there exists $c_{1}>0$, such that, on the $\left(\lambda,\|u\|_{\infty}\right)$-plane, (i) when $0<c<c_{1}$, the bifurcation curve is $S$-shaped; (ii) when $c \geq c_{1}$, the bifurcation curve is $\subset$-shaped. Our results can be applied to the one-dimensional perturbed Gelfand equation with $f(u)=\exp \left(\frac{a u}{a+u}\right)$ for $a \geq 4.37$.
\end{abstract}

\section{Introduction}

In this paper, we study the classification and evolution of bifurcation curves of positive solutions for the one-dimensional Dirichlet-Neumann boundary value problem

$$
\left\{\begin{array}{l}
u^{\prime \prime}(x)+\lambda f(u)=0, \quad 0<x<1, \\
u(0)=0, \quad u^{\prime}(1)=-c<0
\end{array}\right.
$$

where $\lambda>0$ is a bifurcation parameter and the value $c>0$, with which $-c$ is the boundary slope of $u(x)$ at $x=1$, is treated as an evolution parameter. We assume that nonlinearity $f \in C^{2}[0, \infty)$ satisfies the following hypotheses (H1)-(H6):

(H1) $f(u), f^{\prime}(u)>0$ on $[0, \infty)$.

Received October 7, 2017; Accepted April 10, 2018.

Communicated by Cheng-Hsiung Hsu.

2010 Mathematics Subject Classification. 34B18, 74G35.

Key words and phrases. bifurcation, multiplicity, positive solution, $S$-shaped bifurcation curve, $\subset$-shaped bifurcation curve, time map.

This work is partially supported by the Ministry of Science and Technology of the Republic of China under grant No. MOST 103-2115-M-007-001-MY2.

*Corresponding author. 
(H2) $f$ is convex-concave on $(0, \infty)$; that is, there exists a number $\gamma>0$ such that

$$
f^{\prime \prime}(u) \begin{cases}>0 & \text { when } u \in[0, \gamma) \\ =0 & \text { when } u=\gamma \\ <0 & \text { when } u \in(\gamma, \infty)\end{cases}
$$

In addition, $\gamma^{2} f(\gamma) \geq 3 \int_{0}^{\gamma} t f(t) d t$.

(H3) $f$ is asymptotic sublinear at infinity; that is, $\lim _{u \rightarrow \infty} f(u) / u=0$.

(H4) There exists a number $\tau>0$ such that

$$
\left[f^{\prime}(u)+u f^{\prime \prime}(u)\right] f(u)-u\left[f^{\prime}(u)\right]^{2} \begin{cases}>0 & \text { when } u \in[0, \tau) \\ =0 & \text { when } u=\tau \\ <0 & \text { when } u \in(\tau, \infty)\end{cases}
$$

(H5) $\left[f^{\prime}(u)\right]^{2}-f^{\prime \prime}(u) f(u)>0$ on $[0, \infty)$.

(H6) Define

(1.6) $N_{2}(u)=N_{1}(u)-3 M_{2}(u)-2, \quad N_{3}(u)=-2 N_{1}(u)+3 M_{2}(u)+3$ for $u>0$,

$$
\begin{gathered}
P_{1}(u, s)=\frac{u f(u)-s f(s)}{F(u)-F(s)}, \quad P_{2}(u, s)=\frac{u^{2} f^{\prime}(u)-s^{2} f^{\prime}(s)}{u f(u)-s f(s)} \quad \text { for } 0 \leq s<u, \\
W(u, s)=P_{1}(u, s)\left[N_{2}(u)+2 P_{2}(u, s)\right] \quad \text { for } 0 \leq s<u, \\
\widetilde{W}_{0}(u)=\left[\frac{\partial}{\partial s} W(u, s)\right]_{s=0} \quad \text { for } u>0 .
\end{gathered}
$$

Then

$$
f(\tau) \geq 4 f(0)
$$

and the following three inequalities related to $W(u, s)$ hold:

$$
\begin{gathered}
\widetilde{W}_{0}(u)>0 \quad \text { for } 0<u \leq \bar{\rho}, \\
W(u, s) \geq W(u, 0)+s \widetilde{W}_{0}(u) \quad \text { for } 0 \leq s<u \leq \bar{\rho}, \\
3 \sqrt{f(u)}\left[W(u, 0)+N_{3}(u)\right]+2 u \sqrt{f(0)} \widetilde{W}_{0}(u)>0 \quad \text { for } 0<u \leq \bar{\rho},
\end{gathered}
$$


where $\bar{\rho}$ is the unique positive zero of

$$
2 f(0)\left[2-M_{1}(u)\right]-f(u)
$$

on $(0, \tau)$.

Remark 1.1. The existence and uniqueness of the number $\bar{\rho}$ in $(0, \tau)$ in $(\mathrm{H} 6)$ is proved in Lemma 3.1(ix) stated behind.

The motivation of this paper arises from the recent work of Liang and Wang [11]. In 11, the authors considered the classification and evolution of bifurcation curves of positive solutions for the one-dimensional perturbed Gelfand equation with Dirichlet-Neumann boundary conditions, i.e., (1.1) with $f(u)=\exp \left(\frac{a u}{a+u}\right), a>0$. In this paper, we generalize their main results to general nonlinearities $f(u)$ under hypotheses (H1)-(H6).

For one-dimensional zero Dirichlet boundary value problem with general nonlinearity:

$$
\left\{\begin{array}{l}
u^{\prime \prime}(x)+\lambda f(u)=0, \quad 0<x<1, \\
u(0)=u(1)=0
\end{array}\right.
$$

the shapes of the bifurcation curve of positive solutions for $(1.14)$ on the $\left(\lambda,\|u\|_{\infty}\right)$-plane have been studied exuberantly; see, e.g., [1, 5, 6, 8, 10] and references therein. While the shapes of the bifurcation curves with the mixed boundary conditions such as (1.1) are much less studied; see [2, 4, 7, 11]. We define the bifurcation curve of positive solutions of (1.1) by

$$
\left.\widetilde{S}_{c}=\left\{\left(\lambda,\left\|u_{\lambda}\right\|_{\infty}\right): \lambda>0 \text { and } u_{\lambda} \text { is a positive solution of } 1.1\right\}\right\},
$$

while that of 1.14 is defined by

$$
\left.S=\left\{\left(\lambda,\left\|u_{\lambda}\right\|_{\infty}\right): \lambda>0 \text { and } u_{\lambda} \text { is a positive solution of } 1.14\right)\right\}
$$

Moreover, we say that, on the $\left(\lambda,\|u\|_{\infty}\right)$-plane, the shape of a bifurcation curve $\widetilde{S}_{c}$ (same for $S$ ) is $S$-shaped or $\subset$-shaped if it satisfies the following conditions, respectively.

$S$-shaped. The bifurcation curve $\widetilde{S}_{c}$ on the $\left(\lambda,\|u\|_{\infty}\right)$-plane is said to be $S$-shaped if $\widetilde{S}_{c}$ has at least two turning points, say $\left(\lambda^{*},\left\|u_{\lambda^{*}}\right\|_{\infty}\right)$ and $\left(\lambda_{*},\left\|u_{\lambda_{*}}\right\|_{\infty}\right)$, satisfying $\lambda_{*}<\lambda^{*}$ and $\left\|u_{\lambda^{*}}\right\|_{\infty}<\left\|u_{\lambda_{*}}\right\|_{\infty}$, and

(i) $\widetilde{S}_{c}$ starts at some point $\left(\lambda_{0},\left\|u_{\lambda_{0}}\right\|_{\infty}\right)$ and initially continues to the right,

(ii) at $\left(\lambda^{*},\left\|u_{\lambda^{*}}\right\|_{\infty}\right), \widetilde{S}_{c}$ turns to the left,

(iii) at $\left(\lambda_{*},\left\|u_{\lambda_{*}}\right\|_{\infty}\right), \widetilde{S}_{c}$ turns to the right,

(iv) $\widetilde{S}_{c}$ tends to infinity as $\lambda \rightarrow \infty$. That is, $\lim _{\lambda \rightarrow \infty}\left\|u_{\lambda}\right\|_{\infty}=\infty$. 
Exactly $S$-shaped. The bifurcation curve $\widetilde{S}_{c}$ on the $\left(\lambda,\|u\|_{\infty}\right)$-plane is said to be exactly $S$-shaped if $\widetilde{S}_{c}$ is $S$-shaped and it has exactly two turning points; see Figure 1.1.

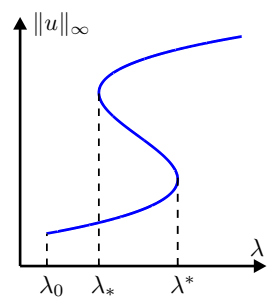

(i)

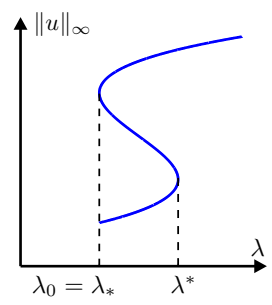

(ii)

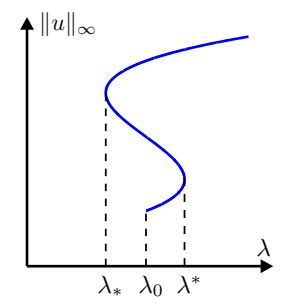

(iii)

Figure 1.1: Three different types of exactly $S$-shaped bifurcation curves $\widetilde{S}_{c}$ with $\lambda_{0}>0$ and $\left\|u_{\lambda_{0}}\right\|_{\infty}>0$. (i) Type 1 with $\lambda_{0}<\lambda_{*}$. (ii) Type 2 with $\lambda_{0}=\lambda_{*}$. (iii) Type 3 with $\lambda_{0}>\lambda_{*}$.

Type 1/2/3 $S$-shaped. Assume that the bifurcation curve $\widetilde{S}_{c}$ is $S$-shaped on the $(\lambda$, $\left.\|u\|_{\infty}\right)$-plane. Let $\left(\lambda_{0},\left\|u_{\lambda_{0}}\right\|_{\infty}\right)$ be the starting point of $\widetilde{S}_{c}$, and

$$
\bar{\lambda}_{\min } \equiv \min \left\{\lambda:\left(\lambda,\left\|u_{\lambda}\right\|_{\infty}\right) \text { is a turning point of } \widetilde{S}_{c}\right\}
$$

Then $\widetilde{S}_{c}$ is said to be type 1 (resp., type 2 and type 3) $S$-shaped if $\lambda_{0}<\bar{\lambda}_{\text {min }}$ (resp., $\lambda_{0}=\bar{\lambda}_{\min }$ and $\lambda_{0}>\bar{\lambda}_{\text {min }}$ ); see Figure 1.1(i) (resp., Figures 1.1(ii) and 1.1(iii)).

$\subset$-shaped. The bifurcation curve $\widetilde{S}_{c}$ on the $\left(\lambda,\|u\|_{\infty}\right)$-plane is said to be $\subset$-shaped if $\widetilde{S}_{c}$ has at least one turning point $\left(\lambda_{*},\left\|u_{\lambda_{*}}\right\|_{\infty}\right)$, and

(i) $\widetilde{S}_{c}$ starts at some point $\left(\lambda_{0},\left\|u_{\lambda_{0}}\right\|_{\infty}\right)$ and initially continues to the left,

(ii) at $\left(\lambda_{*},\left\|u_{\lambda_{*}}\right\|_{\infty}\right), \widetilde{S}_{c}$ turns to the right,

(iii) $\lambda_{*}<\lambda_{0}$ and $\left\|u_{\lambda_{0}}\right\|_{\infty}<\left\|u_{\lambda_{*}}\right\|_{\infty}$,

(iv) $\widetilde{S}_{c}$ tends to infinity as $\lambda \rightarrow \infty$. That is, $\lim _{\lambda \rightarrow \infty}\left\|u_{\lambda}\right\|_{\infty}=\infty$.

Exactly $\subset$-shaped. The bifurcation curve $\widetilde{S}_{c}$ on the $\left(\lambda,\|u\|_{\infty}\right)$-plane is said to be exactly $\subset$-shaped if $\widetilde{S}_{c}$ is $\subset$-shaped and it has exactly one turning point; see Figure 1.2 .

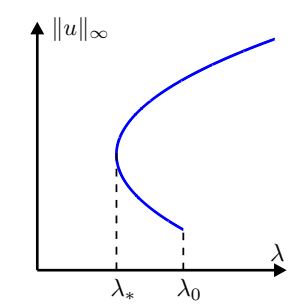

Figure 1.2: Exactly $\subset$-shaped bifurcation curve $\widetilde{S}_{c}$ with $\lambda_{0}>0$ and $\left\|u_{\lambda_{0}}\right\|_{\infty}>0$. 
For one-dimensional zero Dirichlet boundary value problem (1.14), under (H1)-(H3) on $f$, Hung and Wang [6] proved that the bifurcation curve of positive solutions is exactly type $1 S$-shaped on the $\left(\lambda,\|u\|_{\infty}\right)$-plane. They gave an application to the one-dimensional perturbed Gelfand problem.

Theorem 1.2. 6, Theorems 2.1(i) and 2.2(i)] Consider 1.14 and suppose that nonlinearity $f \in C^{2}[0, \infty)$ satisfies (H1)-(H3). Then, on the $\left(\lambda,\|u\|_{\infty}\right)$-plane, the bifurcation curve $S$ is a continuous curve which starts at the origin and tends to infinity as $\lambda \rightarrow \infty$. Moreover, it is exactly type $1 S$-shaped. In particular, $f(u)=\exp \left(\frac{a u}{a+u}\right)$ satisfies $(\mathrm{H} 1)-$ (H3) for $a \geq a^{*} \approx 4.166$ for some $a^{*}$ defined in [6, Eq. (3.22)].

For one-dimensional Dirichlet-Neumann boundary value problem (1.1) with $f(u)=$ $\exp \left(\frac{a u}{a+u}\right)$, Goddard II, Shivaji and Lee 3, Section 3.4] started to consider with $c=1$. Their computational results suggested that there exists a positive critical bifurcation value $a^{* *}<4$ such that $\widetilde{S}_{1}$ is strictly increasing for $0<a \leq a^{* *}$ and is exactly $S$-shaped for $a>a^{* *}$. Hung, Wang and $\mathrm{Yu}[7]$ gave rigorous proofs for some of these computational results. Recently, Liang and Wang [11] generalized these analytic results to general $c>0$. The main result in 11 is stated in the next theorem.

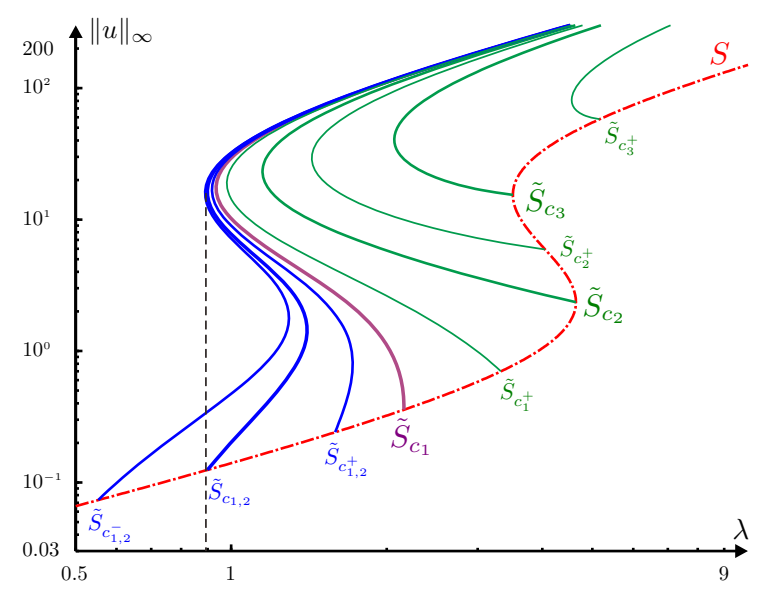

Figure 1.3: (cf. [11, Figure 4]) Numerical simulations of bifurcation curves $S$ and $\widetilde{S}_{c}$ for $f(u)=\exp \left(\frac{a u}{a+u}\right)$ with $a=5$ and varying $c>0$ on the $\left(\lambda,\left\|u_{\lambda}\right\|_{\infty}\right)$-plane of the bi-logarithm coordinates. Here $0<c_{1,2}^{-}<c_{1,2}(\approx 0.49)<c_{1,2}^{+}<c_{1}(\approx 1.36)<c_{1}^{+}<c_{2}(\approx 7.72)<c_{2}^{+}<$ $c_{3}(\approx 47.71)<c_{3}^{+}$.

Theorem 1.3. (cf. [11, Theorem 2.3], see Figure1.3 with $a=5$ ) Consider (1.1] with $c>0$ and $f(u)=\exp \left(\frac{a u}{a+u}\right)$ for any fixed $a>0$. Then, on the $\left(\lambda,\|u\|_{\infty}\right)$-plane, the bifurcation curve $\widetilde{S}_{c}$ is a continuous curve which starts at some point $\left(\lambda_{0},\left\|u_{\lambda_{0}}\right\|_{\infty}\right)$ with $\lambda_{0}>0$ and $\left\|u_{\lambda_{0}}\right\|_{\infty}>0$ and it tends to infinity as $\lambda \rightarrow \infty$. Moreover, when $a \geq a_{1}(\approx 4.107)$, where 
$a_{1}$ is defined in [6. Eq. (1.4)], there exists $c_{1}\left(=c_{1}(a)\right)$ such that the following assertions (a)-(b) hold:

(a) For $0<c<c_{1}$, the bifurcation curve $\widetilde{S}_{c}$ is $S$-shaped on the $\left(\lambda,\|u\|_{\infty}\right)$-plane. More precisely, there exist three positive $c_{1,1} \leq c_{1,2} \leq c_{1,3}$ on $\left(0, c_{1}\right)$, all depending on $a$, such that the $S$-shaped belongs to type 1 , type 2 and type 3 when $0<c<c_{1,1}, c=c_{1,2}$ and $c_{1,3}<c<c_{1}$, respectively.

(b) For $c \geq c_{1}$, the bifurcation curve $\widetilde{S}_{c}$ is $\subset$-shaped on the $\left(\lambda,\|u\|_{\infty}\right)$-plane.

The paper is organized as follows. Section 2 contains statements of the main results (Theorems 2.1, 2.3 2.5; in particular, Theorems 2.4 and 2.5). Section 3 contains several lemmas needed to prove the main results and their proofs except those of Lemma 3.1(i)(ii), (ix)-(x) and Lemma 3.7. Section 4 contains proofs of the main results except assertions (a)-(d) stated in the proof of Theorem 2.5 for the function $f(\rho)=\exp \left(\frac{a \rho}{a+\rho}\right)$ with $a \geq 4.37$. (The proofs of Lemma 3.1(i)-(ii), (ix)-(x) and Lemma 3.7 and assertions (a)-(d) stated in the proof of Theorem 2.5 are put in [9] due to their lengthiness.)

\section{Main results}

The main results are next Theorems 2.1, 2.3 2.5, in particular, Theorems 2.4 and 2.5 .

Theorem 2.1. (cf. Figure 1.3 for $f(u)=\exp (a u /(a+u))$ with $a=5)$ Consider (1.1) with $c>0$ and suppose that $f$ satisfies $(\mathrm{H} 1)$ and $(\mathrm{H} 3)-(\mathrm{H} 5)$. Then, on the $\left(\lambda,\|u\|_{\infty}\right)$-plane, the bifurcation curve $\widetilde{S}_{c}$ is a continuous curve which starts at some point $\left(\lambda_{0},\left\|u_{\lambda_{0}}\right\|_{\infty}\right)$ with $\lambda_{0}\left(=\lambda_{0}(c)\right)>0$ and $\left\|u_{\lambda_{0}}\right\|_{\infty}>0$. More precisely, the following assertions (i)-(iv) hold:

(i) $S \cap \widetilde{S}_{c}=\left\{\left(\lambda_{0},\left\|u_{\lambda_{0}}\right\|_{\infty}\right)\right\}$. Moreover, if $u_{\lambda}$ is a positive solution of (1.1) with $u_{\lambda} \neq$ $u_{\lambda_{0}}$, then $\left\|u_{\lambda}\right\|_{\infty}>\left\|u_{\lambda_{0}}\right\|_{\infty}$.

(ii) $\widetilde{S}_{c}$ tends to infinity as $\lambda \rightarrow \infty$. That is, $\lim _{\lambda \rightarrow \infty}\left\|u_{\lambda}\right\|_{\infty}=\infty$.

(iii) For any $\rho>\rho_{0}(c) \equiv\left\|u_{\lambda_{0}(c)}\right\|_{\infty}>0$, there exist exactly two positive $\widetilde{\lambda}(\rho)<\lambda(\rho)$ such that $(\widetilde{\lambda}(\rho), \rho) \in \widetilde{S}_{c}$ and $(\lambda(\rho), \rho) \in S$.

(iv) $\rho_{0}(c) \in C(0, \infty)$ is a strictly increasing function of $c$ on $(0, \infty), \lim _{c \rightarrow 0^{+}} \rho_{0}(c)=0$ and $\lim _{c \rightarrow \infty} \rho_{0}(c)=\infty$.

Remark 2.2. (cf. Figure 1.3 for $f(u)=\exp \left(\frac{a u}{a+u}\right)$ with $a=5$ ) Theorem 1.2 together with Theorem 2.1(iv) imply that, if $f$ satisfies (H1)-(H5), then there exist two positive numbers $c_{2}<c_{3}$ such that bifurcation curves $S$ and $\widetilde{S}_{c}$ intersect at the lower (resp., middle and upper) branch of exactly type $1 S$-shaped bifurcation curve $S$ when $c \in\left(0, c_{2}\right)$ (resp., $c \in\left(c_{2}, c_{3}\right)$ and $\left.c \in\left(c_{3}, \infty\right)\right)$. 
Theorem 2.3. (cf. Figure 1.3 for $f(u)=\exp \left(\frac{a u}{a+u}\right)$ with $\left.a=5\right)$ Consider 1.1) with $c>0$ and suppose that $f$ satisfies (H1) and (H3)-(H5). Then the following assertions (i) and (ii) hold:

(i) For any two positive numbers $\widetilde{c}_{1}<\widetilde{c}_{2}, \widetilde{S}_{\widetilde{c}_{1}}$ lies at the left-hand side of $\widetilde{S}_{\widetilde{c}_{2}}$ on the $\left(\lambda,\|u\|_{\infty}\right)$-plane. That is, for any two positive numbers $\widetilde{c}_{1}<\widetilde{c}_{2}$ and $\rho>\rho_{0}\left(\widetilde{c}_{2}\right)$, let $\left(\lambda_{\widetilde{c}_{i}}(\rho), \rho\right) \in \widetilde{S}_{\widetilde{c}_{i}}, i=1,2$. Then $\lambda_{\widetilde{c}_{1}}(\rho)<\lambda_{\widetilde{c}_{2}}(\rho)$.

(ii) Let $\lambda_{\min }(c) \equiv \min \left\{\lambda:\left(\lambda,\left\|u_{\lambda}\right\|_{\infty}\right) \in \widetilde{S}_{c}\right\}$. Then $\lambda_{\min }(c)$ is strictly increasing on $(0, \infty), \lim _{c \rightarrow 0^{+}} \lambda_{\min }(c)=0$ and $\lim _{c \rightarrow \infty} \lambda_{\min }(c)=\infty$.

Theorem 2.4. (cf. Figure 1.3 for $f(u)=\exp \left(\frac{a u}{a+u}\right)$ with $\left.a=5\right)$ Consider (1.1) with $c>0$ and suppose that $f$ satisfies $(\mathrm{H} 1)-(\mathrm{H} 6)$. Then there exists a unique positive $c_{1}$ such that the following assertions (i)-(ii) hold:

(i) For $0<c<c_{1}$, the bifurcation curve $\widetilde{S}_{c}$ is $S$-shaped on the $\left(\lambda,\|u\|_{\infty}\right)$-plane. More precisely, there exist three positive $c_{1,1} \leq c_{1,2} \leq c_{1,3}$ on $\left(0, c_{1}\right)$ such that the following assertions (a)-(c) hold:

(a) (cf. Figure 1.1(i)) If $0<c<c_{1,1}$, then the bifurcation curve $\widetilde{S}_{c}$ is type 1 $S$-shaped on the $\left(\lambda,\|u\|_{\infty}\right)$-plane. Moreover, there exist three positive $\lambda_{0}<$ $\lambda_{*}<\lambda^{*}$ which are all strictly increasing functions of $c$ on $\left(0, c_{1,1}\right)$ such that (1.1) has no positive solution for $0<\lambda<\lambda_{0}$, at least one positive solution for $\lambda_{0} \leq \lambda<\lambda_{*}$ and $\lambda>\lambda^{*}$, at least two positive solutions for $\lambda=\lambda_{*}$ and $\lambda=\lambda^{*}$, and at least three positive solutions for $\lambda_{*}<\lambda<\lambda^{*}$.

(b) (cf. Figure 1.1(ii)) If $c=c_{1,2}$, then the bifurcation curve $\widetilde{S}_{c}$ is type $2 S$-shaped on the $\left(\lambda,\|u\|_{\infty}\right)$-plane. Moreover, there exist three positive $\lambda_{0}=\lambda_{*}<\lambda^{*}$ such that (1.1) has no positive solution for $0<\lambda<\lambda_{0}$, at least one positive solution for $\lambda>\lambda^{*}$, at least two positive solutions for $\lambda=\lambda_{*}$ and $\lambda=\lambda^{*}$, and at least three positive solutions for $\lambda_{*}<\lambda<\lambda^{*}$.

(c) (cf. Figure 1.1(iii)) If $c_{1,3}<c<c_{1}$, then the bifurcation curve $\widetilde{S}_{c}$ is type $3 S$ shaped on the $\left(\lambda,\|u\|_{\infty}\right)$-plane. Moreover, there exist three positive $\lambda_{*}<\lambda_{0}<$ $\lambda^{*}$ which are all strictly increasing functions of $c$ on $\left(c_{1,3}, c_{1}\right)$ such that $(1.1)$ has no positive solution for $0<\lambda<\lambda_{*}$, at least one positive solution for $\lambda=\lambda_{*}$ and $\lambda>\lambda^{*}$, at least two positive solutions for $\lambda_{*}<\lambda<\lambda_{0}$ and $\lambda=\lambda^{*}$, and at least three positive solutions for $\lambda_{0} \leq \lambda<\lambda^{*}$.

(ii) (cf. Figure 1.2) For $c \geq c_{1}$, the bifurcation curve $\widetilde{S}_{c}$ is $\subset$-shaped on the $\left(\lambda,\|u\|_{\infty}\right)$ plane. Moreover, there exist two positive $\lambda_{*}<\lambda_{0}$ such that (1.1) has no positive solution for $0<\lambda<\lambda_{*}$, at least one positive solution for $\lambda=\lambda_{*}$ and $\lambda>\lambda_{0}$, and at least two positive solutions for $\lambda_{*}<\lambda \leq \lambda_{0}$. 
Theorem 2.5. (cf. Figure 1.3 with $a=5)$ Consider 1.1 for $f(u)=\exp \left(\frac{a u}{a+u}\right)$ with $a \geq 4.37$. Then $f$ satisfies (H1)-(H6) with $\gamma=a(a-2) / 2>\tau=a$ and hence all results in Theorems 2.1, 2.3 and 2.4 hold.

\section{Lemmas}

To prove our main results for problem (1.1), in this paper, we modify the quadrature method (time-map technique) which was used in 3, 4, 7, 11]. First, the time map formula which we apply to study for zero Dirichlet problem $(1.14)$ takes the form as follows:

$$
G(\rho) \equiv \sqrt{2} \int_{0}^{\rho} \frac{d s}{\sqrt{F(\rho)-F(s)}} \text { for } \rho>0
$$

where $F(s)=\int_{0}^{s} f(t) d t$ is defined in 1.2 . Note that positive solutions $u$ of (1.14) correspond to

$$
\|u\|_{\infty}=\rho \quad \text { and } \quad G(\rho)=\sqrt{\lambda}
$$

Thus, studying the exact number of positive solutions of 1.14 is equivalent to studying the shape of the time map $G(\rho)$ on $(0, \infty)$. We compute that

$$
G^{\prime}(\rho)=\frac{\sqrt{2}}{2} \int_{0}^{\rho} \frac{\theta(\rho)-\theta(s)}{\rho[F(\rho)-F(s)]^{3 / 2}} d s,
$$

where $\theta(s) \equiv 2 F(s)-s f(s)$. Moreover,

$$
G^{\prime \prime}(\rho)=\frac{\sqrt{2}}{2} \int_{0}^{\rho} \frac{\frac{3}{2}\left[P_{1}(\rho, s)\right]^{2}-\left[P_{2}(\rho, s)+2\right] P_{1}(\rho, s)}{\rho^{2}[F(\rho)-F(s)]^{1 / 2}} d s,
$$

where $P_{1}(\rho, s)$ and $P_{2}(\rho, s)$ are defined in (1.7); see [1, Eq. (2.7)]. Note that

$$
\lim _{\rho \rightarrow 0^{+}} G(\rho)=0 \text { and } \lim _{\rho \rightarrow \infty} G(\rho)=\infty
$$

if $f \in C^{2}[0, \infty)$ satisfies (H1) and (H3); see, e.g., 6, Lemma 3.1].

On the other hand, considering Dirichlet-Neumann problem (1.1), we define

$$
\begin{aligned}
\widetilde{H}_{c}(\rho, q) & =2 \int_{0}^{\rho} \frac{d s}{\sqrt{F(\rho)-F(s)}}-\int_{0}^{q} \frac{d s}{\sqrt{F(\rho)-F(s)}}-\frac{c}{\sqrt{F(\rho)-F(q)}} \\
& =\sqrt{2} G(\rho)-\int_{0}^{q} \frac{d s}{\sqrt{F(\rho)-F(s)}}-\frac{c}{\sqrt{F(\rho)-F(q)}}
\end{aligned}
$$

for $0 \leq q<\rho<\infty$; see 3 . Eq. (3.29)] for $f(\rho)=\exp \left(\frac{a \rho}{a+\rho}\right)$ and $c=1$. Then to study the number of positive solutions of $(1.1)$, we need to analyze function $\widetilde{H}_{c}(\rho, q)$ in the first step. Beforehand, under (H1) and (H3)-(H5), we derive some basic properties related to functions $f(\rho), f^{\prime}(\rho)$ and $F(\rho)$ in Lemma 3.1 to ease the proofs of the other lemmas in this section. 
Lemma 3.1. Suppose that $f \in C^{2}[0, \infty)$ satisfies (H1) and (H3)-(H5), and let $M_{1}(\rho)$, $M_{2}(\rho), P_{1}(\rho, s)$ and $P_{2}(\rho, s)$ be defined in (1.3), (1.4) and (1.7). Then the following assertions (i)-(x) hold:

(i) $M_{2}(\rho)<M_{1}(\rho)$ for $\rho>0$.

(ii) Let $\tau>0$ be defined in (H4). Then

$$
M_{2}^{\prime}(\rho) \begin{cases}>0 & \text { when } \rho \in[0, \tau) \\ =0 & \text { when } \rho=\tau \\ <0 & \text { when } \rho \in(\tau, \infty)\end{cases}
$$

(iii) $P_{1}(\rho, 0)=M_{1}(\rho), \lim _{s \rightarrow \rho^{-}} P_{1}(\rho, s)=M_{2}(\rho)+1$ for $\rho>0$, and $\lim _{\rho \rightarrow s^{+}} P_{1}(\rho, s)=$ $M_{2}(s)+1$ for $s \geq 0$.

(iv) $P_{1}(\rho, s)>1$ for $0 \leq s<\rho$.

(v) For $0<\rho \leq \tau, P_{1}(\rho, s)$ is a strictly increasing function of $s$ on $[0, \rho)$. Moreover, $M_{1}(\rho) \leq P_{1}(\rho, s)<M_{2}(\rho)+1$ for $0 \leq s<\rho \leq \tau$, where the equality holds if and only if $s=0$.

(vi) $M_{2}(\rho)<P_{2}(\rho, s)$ for $0<s<\rho \leq \tau$.

(vii) The function $Q(\rho, s) \equiv \theta(\rho)-\theta(s)+M_{1}(\rho)[F(\rho)-F(s)]>0$ for $0 \leq s<\rho$.

(viii) $\left[\frac{f(\rho)}{\sqrt{F(\rho)}}\right]^{\prime}+\frac{M_{2}(\rho)+1}{2 \rho} \frac{f(\rho)}{\sqrt{F(\rho)}}>0$ for $0<\rho \leq \tau$.

(ix) If $f$ satisfies (1.10), then there exists a unique $\bar{\rho}$ on $(0, \tau)$ such that

$$
2 f(0)\left[2-M_{1}(\rho)\right]-f(\rho) \begin{cases}>0 & \text { when } \rho \in(0, \bar{\rho}) \\ =0 & \text { when } \rho=\bar{\rho} \\ <0 & \text { when } \rho \in(\bar{\rho}, \tau]\end{cases}
$$

(x) If $f$ satisfies 1.10 , then the function

$$
R(\rho, s) \equiv 2 f(0)[\theta(\rho)-\theta(s)]-\frac{f(\rho)}{\sqrt{F(\rho)}} \sqrt{\rho} \frac{[F(\rho)-F(s)]^{3 / 2}}{\sqrt{\rho-s}} \quad \text { for } 0 \leq s<\rho
$$

satisfies $R(\rho, s)<0$ for $0 \leq s<\rho$ and $\rho>\bar{\rho}$.

The proofs of Lemma 3.1(iii)-(viii) are very similar to those of [11, Lemma 3.1], and hence we omit them here. While the proofs of the remaining parts (i)-(ii) and (ix)-(x) are easy but tedious, and hence we put them in [9]. 
Lemma 3.2. Suppose that $f \in C^{2}[0, \infty)$ satisfies $(\mathrm{H} 1)$ and $(\mathrm{H} 3)-(\mathrm{H} 5)$. Then, for $\widetilde{H}_{c}(\rho, q)$ with $0 \leq q<\rho<\infty$ and $c>0$, the following assertions (i)-(viii) hold:

(i) For $c>0, \lim _{\rho \rightarrow 0^{+}} \widetilde{H}_{c}(\rho, 0)=-\infty$ and $\lim _{\rho \rightarrow \infty} \widetilde{H}_{c}(\rho, 0)=\infty$.

(ii) For $c>0$, there exists a unique positive $\rho_{0}\left(=\rho_{0}(c)\right)$ such that

$$
\widetilde{H}_{c}(\rho, 0) \begin{cases}<0 & \text { when } \rho \in\left(0, \rho_{0}\right) \\ =0 & \text { when } \rho=\rho_{0} \\ >0 & \text { when } \rho \in\left(\rho_{0}, \infty\right)\end{cases}
$$

(iii) For fixed $c, \rho>0, \widetilde{H}_{c}(\rho, q)$ is a strictly decreasing function of $q$ on $[0, \rho)$ and $\lim _{q \rightarrow \rho^{-}} \widetilde{H}_{c}(\rho, q)=-\infty$.

(iv) For $c>0$, if $0<\rho<\rho_{0}(c)$, then $\widetilde{H}_{c}(\rho, q)$ has no zero $q(=q(\rho, c))$ on $[0, \rho)$, while if $\rho \geq \rho_{0}(c)$, then $\widetilde{H}_{c}(\rho, q)$ has a unique zero $q(\rho, c)$ on $[0, \rho)$, i.e.,

$$
\widetilde{H}_{c}(\rho, q(\rho, c))=0 .
$$

Moreover, $q(\rho, c)=0$ if and only if $\rho=\rho_{0}(c)$.

(v) For $c>0$ and $\rho>\rho_{0}, q(\rho, c) \in C\left[\rho_{0}, \infty\right) \cap C^{1}\left(\rho_{0}, \infty\right)$ satisfies

$$
\frac{\partial}{\partial \rho} q(\rho, c)=\frac{[F(\rho)-F(q(\rho, c))]^{3 / 2}\left[2 \sqrt{2} G^{\prime}(\rho)+\int_{0}^{q(\rho, c)} \frac{f(\rho)}{[F(\rho)-F(s)]^{3 / 2}} d s\right]+c f(\rho)}{2[F(\rho)-F(q(\rho, c))]+c f(q(\rho, c))} .
$$

Moreover,

$$
\lim _{\rho \rightarrow \rho_{0}^{+}} \frac{\partial}{\partial \rho} q(\rho, c)=\frac{2 \sqrt{2}\left[F\left(\rho_{0}\right)\right]^{3 / 2} G^{\prime}\left(\rho_{0}\right)+c f\left(\rho_{0}\right)}{2 F\left(\rho_{0}\right)+c f(0)}
$$

(vi) For $c>0$ and $\rho \geq \rho_{0}$,

$$
0<\rho-q(\rho, c) \leq \frac{c^{2} f(\rho)}{4 f(0) \rho}
$$

Moreover, $\lim _{\rho \rightarrow \infty}[\rho-q(\rho, c)]=0$.

(vii) $\rho_{0}(c)$ is a continuous, strictly increasing function of $c$ on $(0, \infty), \lim _{c \rightarrow 0^{+}} \rho_{0}(c)=0$ and $\lim _{c \rightarrow \infty} \rho_{0}(c)=\infty$. Moreover, for $c>0, \rho_{0}(c)$ is the unique positive root of

$$
c=\sqrt{2 F(\rho)} G(\rho) .
$$

(viii) For $\rho>0, q(\rho, c) \in C(0, \widehat{c}] \cap C^{1}(0, \widehat{c})$ is a strictly decreasing function of $c$ on $(0, \widehat{c}]$, $\lim _{c \rightarrow 0^{+}} q(\rho, c)=\rho$ and $q(\rho, \widehat{c})=0$. Here $\widehat{c}(=\widehat{c}(\rho))=\sqrt{2 F(\rho)} G(\rho)$. 
Proof. Lemma 3.2(iii) and (iv) are slight generalizations of 3 , Lemma B and Theorem 3.4(a)], and Lemma 3.2(ii), (vii) and (viii) are from [11, Lemma 3.2(ii), (vii) and (viii)], respectively. Hence these proofs are omitted. We then prove Lemma 3.2(i), (v) and (vi) as follows.

(i) Since $f \in C^{2}[0, \infty)$ satisfies (H1) and (H3), the two limits in (3.4) hold by 6 , Lemma 3.1]. Then, by (3.5), we have that

$$
\begin{aligned}
& \lim _{\rho \rightarrow 0^{+}} \widetilde{H}_{c}(\rho, 0)=\lim _{\rho \rightarrow 0^{+}}\left[\sqrt{2} G(\rho)-\frac{c}{\sqrt{F(\rho)}}\right]=-\infty, \\
& \lim _{\rho \rightarrow \infty} \widetilde{H}_{c}(\rho, 0)=\lim _{\rho \rightarrow \infty}\left[\sqrt{2} G(\rho)-\frac{c}{\sqrt{F(\rho)}}\right]=\infty .
\end{aligned}
$$

So Lemma 3.2(i) holds.

(v) Since $\widetilde{H}_{c}(\rho, q(\rho, c))=0$ by $(3.7)$ and applying the Implicit Function Theorem to (3.5), we have that

$$
\begin{aligned}
\frac{\partial}{\partial \rho} q(\rho, c) & =-\frac{\frac{\partial}{\partial \rho} \widetilde{H}_{c}(\rho, q(\rho, c))}{\frac{\partial}{\partial q} \widetilde{H}_{c}(\rho, q(\rho, c))} \\
& =\frac{\sqrt{2} G^{\prime}(\rho)+\int_{0}^{q(\rho, c)} \frac{f(\rho)}{2[F(\rho)-F(s)]^{3 / 2}} d s+\frac{c f(\rho)}{2[F(\rho)-F(s)]^{3 / 2}}}{\frac{1}{\sqrt{F(\rho)-F(q(\rho, c))}}+\frac{c f(\rho))}{2[F(\rho)-F(q(\rho, c))]^{3 / 2}}} \\
& =\frac{[F(\rho)-F(q(\rho, c))]^{3 / 2}\left[2 \sqrt{2} G^{\prime}(\rho)+\int_{0}^{q(\rho, c)} \frac{f(\rho)}{[F(\rho)-F(s)]^{3 / 2}} d s\right]+c f(\rho)}{2[F(\rho)-F(q(\rho, c))]+c f(q(\rho, c))} .
\end{aligned}
$$

Moreover,

$$
\lim _{\rho \rightarrow \rho_{0}^{+}} \frac{\partial}{\partial \rho} q(\rho, c)=\frac{\left[F\left(\rho_{0}\right)\right]^{3 / 2}\left[2 \sqrt{2} G^{\prime}\left(\rho_{0}\right)\right]+c f\left(\rho_{0}\right)}{2 F\left(\rho_{0}\right)+c f(0)}
$$

since $q\left(\rho_{0}(c), c\right)=0$ by Lemma 3.2 (iv). The proof of the assertion $q(\rho, c) \in C\left[\rho_{0}, \infty\right) \cap$ $C^{1}\left(\rho_{0}, \infty\right)$ is very similar to that of $[7$, Lemma $3.1(\mathrm{iv})]$, and hence we omit it here. So Lemma $3.2(\mathrm{v})$ holds.

(vi) By the definition of $\widetilde{H}_{c}$ in $(3.5)$, we compute that

$$
\begin{aligned}
\widetilde{H}_{c}(\rho, q(\rho, c)) & =2 \int_{0}^{\rho} \frac{d s}{\sqrt{F(\rho)-F(s)}}-\int_{0}^{q(\rho, c)} \frac{d s}{\sqrt{F(\rho)-F(s)}}-\frac{c}{\sqrt{F(\rho)-F(q(\rho, c))}} \\
& \geq \int_{0}^{\rho} \frac{d s}{\sqrt{F(\rho)-F(s)}}-\frac{c}{\sqrt{F(\rho)-F(q(\rho, c))}} \\
& \geq \frac{1}{\sqrt{f(\rho)}} \int_{0}^{\rho} \frac{d s}{\sqrt{\rho-s}}-\frac{1}{\sqrt{f(q(\rho, c))}} \frac{c}{\sqrt{\rho-q(\rho, c)}}
\end{aligned}
$$

(by the Mean Value Theorem and (H1)) 


$$
\begin{aligned}
& =\frac{1}{\sqrt{f(q(\rho, c))}}\left[2 \sqrt{\frac{\rho}{f(\rho)} f(q(\rho, c))}-\frac{c}{\sqrt{\rho-q(\rho, c)}}\right] \\
& \geq \frac{1}{\sqrt{f(q(\rho, c))}}\left[2 \sqrt{\frac{\rho}{f(\rho)} f(0)}-\frac{c}{\sqrt{\rho-q(\rho, c)}}\right]
\end{aligned}
$$

by $(\mathrm{H} 1)$. Then, since $\widetilde{H}_{c}(\rho, q(\rho, c))=0$ by (3.7), we conclude that $0<\rho-q(\rho, c) \leq$ $\frac{c^{2}}{4 f(0)} \frac{f(\rho)}{\rho}$, and hence $\lim _{\rho \rightarrow \infty}[\rho-q(\rho, c)]=0$ by (H3). So Lemma 3.2 (vi) holds.

The proof of Lemma 3.2 is complete.

The time map formula which we apply to study Dirichlet-Neumann problem (1.1) takes the form as follows:

$$
H_{c}(\rho, q(\rho, c)) \equiv \frac{c^{2}}{2[F(\rho)-F(q(\rho, c))]} \quad \text { for } \rho \geq \rho_{0}(c),
$$

where $q(\rho, c)$ is defined in 3.7 . Note that positive solutions $u$ of 1.1 correspond to

$$
\|u\|_{\infty}=\rho \quad \text { and } \quad H_{c}(\rho, q(\rho, c))=\lambda,
$$

see, e.g., [3, Theorem 3.3] and 11, Eq. (3.27)] for $f(\rho)=\exp \left(\frac{a \rho}{a+\rho}\right)$. Thus, studying the number of positive solutions of (1.1) is equivalent to studying the shape of the time map $H_{c}(\rho, q(\rho, c))$ for $\rho \geq \rho_{0}(c)$.

The next lemma is from [11, Lemma 3.3].

Lemma 3.3. Suppose that $f \in C^{2}[0, \infty)$ satisfies $(\mathrm{H} 1)$ and $(\mathrm{H} 3)-(\mathrm{H} 5)$. Then, for $G(\rho)$ with $\rho>0$ and for $H_{c}(\rho, q(\rho, c))$ with $\rho \geq \rho_{0}$ and $c>0$, the following assertions (i)-(iii) hold:

(i) $H_{c}(\rho, q(\rho, c)) \leq[G(\rho)]^{2}$ for $c>0$ and $\rho \geq \rho_{0}$. The equality holds if and only if $\rho=\rho_{0}$.

(ii) $\lim _{\rho \rightarrow \infty} H_{c}(\rho, q(\rho, c))=\infty$ for $c>0$.

(iii) $H_{c}(\rho, q(\rho, c))>\frac{1}{4}[G(\rho)]^{2}$ for $c>0$ and $\rho \geq \rho_{0}$. In addition, $\lim _{c \rightarrow 0^{+}} H_{c}(\rho, q(\rho, c))=$ $\frac{1}{4}[G(\rho)]^{2}$ for $\rho>0$.

Lemma 3.4. Suppose that $f \in C^{2}[0, \infty)$ satisfies (H1) and (H3)-(H5). Then, for $H_{c}(\rho, q(\rho, c))$ with $\rho \geq \rho_{0}$ and $c>0$, the following assertions (i)-(iii) hold:

(i) For any two positive numbers $\widetilde{c}_{1}<\widetilde{c}_{2}, H_{\widetilde{c}_{1}}\left(\rho, q\left(\rho, \widetilde{c}_{1}\right)\right)<H_{\widetilde{c}_{2}}\left(\rho, q\left(\rho, \widetilde{c}_{2}\right)\right)$ for $\rho \geq$ $\rho_{0}\left(\widetilde{c}_{2}\right)$.

(ii) Let $\lambda_{\min }(c) \equiv \min _{\rho \geq \rho_{0}(c)} H_{c}(\rho, q(\rho, c))$ for $c>0$. Then $\lambda_{\min }(c)$ is a strictly increasing function of $c$ on $(0, \infty), \lim _{c \rightarrow 0^{+}} \lambda_{\min }(c)=0$ and $\lim _{c \rightarrow \infty} \lambda_{\min }(c)=\infty$. 
(iii) If $\lim _{\rho \rightarrow \rho_{0}(\widetilde{c})^{+}} \frac{d}{d \rho} H_{\widetilde{c}}(\rho, q(\rho, \widetilde{c})) \geq 0$, then $\frac{d}{d \rho} H_{c}\left(\rho_{0}(\widetilde{c}), q\left(\rho_{0}(\widetilde{c}), c\right)\right)>0$ for $0<c<\widetilde{c}$.

Proof. Lemma 3.4(i)-(ii) are from [11, Lemma 3.4(i)-(ii)]. Hence these proofs are omitted. We next prove Lemma 3.4(iii).

We compute that, by 3.8 and (3.10,

$$
\begin{aligned}
& \frac{d}{d \rho} H_{c}(\rho, q(\rho, c)) \\
= & -\frac{c^{2}\left[f(\rho)-f(q(\rho, c)) \frac{\partial}{\partial \rho} q(\rho, c)\right]}{2[F(\rho)-F(q(\rho, c))]^{2}} \\
= & \frac{c^{2} f(q(\rho, c))}{2[F(\rho)-F(q(\rho, c))]^{1 / 2}\{2[F(\rho)-F(q(\rho, c))]+c f(q(\rho, c))\}} \Psi(\rho, q(\rho, c)),
\end{aligned}
$$

where

$$
\Psi(\rho, q) \equiv 2 \sqrt{2} G^{\prime}(\rho)-\left[2 \frac{f(\rho)}{f(q) \sqrt{F(\rho)-F(q)}}-\int_{0}^{q} \frac{f(\rho)}{[F(\rho)-F(s)]^{3 / 2}} d s\right] .
$$

Moreover, by $(3.9)$ and the fact that $q\left(\rho_{0}(c), c\right)=0$ by Lemma 3.2(iv), we have that

$$
\lim _{\rho \rightarrow \rho_{0}(c)^{+}} \frac{d}{d \rho} H_{c}(\rho, q(\rho, c))=\frac{c^{2}}{\sqrt{F\left(\rho_{0}\right)}\left[2 F\left(\rho_{0}\right)+c f(0)\right]} \Phi\left(\rho_{0}\right),
$$

where

$$
\Phi(\rho) \equiv \sqrt{2} f(0) G^{\prime}(\rho)-\frac{f(\rho)}{\sqrt{F(\rho)}} .
$$

Hence, if $\lim _{\rho \rightarrow \rho_{0}(\widetilde{c})^{+}} \frac{d}{d \rho} H_{\widetilde{c}}(\rho, q(\rho, \widetilde{c})) \geq 0$ for some $\widetilde{c}>0$, then $\Phi\left(\rho_{0}(\widetilde{c})\right) \geq 0$. It follows that

$$
\begin{aligned}
\Psi\left(\rho_{0}(\widetilde{c}), q\left(\rho_{0}(\widetilde{c}), \widetilde{c}\right)\right) & =\Psi\left(\rho_{0}(\widetilde{c}), 0\right)=2\left[\sqrt{2} G^{\prime}\left(\rho_{0}(\widetilde{c})\right)-\frac{f\left(\rho_{0}(\widetilde{c})\right)}{f(0) \sqrt{F\left(\rho_{0}(\widetilde{c})\right)}}\right] \\
& =\frac{2}{f(0)} \Phi\left(\rho_{0}(\widetilde{c})\right) \geq 0
\end{aligned}
$$

by (3.14) and (3.16). Moreover, we compute that

$$
\frac{\partial}{\partial q} \Psi(\rho, q)=2 \frac{f^{\prime}(q) f(\rho)}{[f(q)]^{2} \sqrt{F(\rho)-F(q)}}>0
$$

by $(\mathrm{H} 1)$, which implies that $\Psi\left(\rho_{0}(\widetilde{c}), q\left(\rho_{0}(\widetilde{c}), c\right)\right)>\Psi\left(\rho_{0}(\widetilde{c}), q\left(\rho_{0}(\widetilde{c}), \widetilde{c}\right)\right) \geq 0$ for $0<$ $c<\widetilde{c}$ since $q\left(\rho_{0}(\widetilde{c}), c\right)>q\left(\rho_{0}(\widetilde{c}), \widetilde{c}\right)$ for $0<c<\widetilde{c}$ by Lemma 3.2(viii). Therefore $\frac{d}{d \rho} H_{c}\left(\rho_{0}(\widetilde{c}), q\left(\rho_{0}(\widetilde{c}), c\right)\right)>0$ by 3.13$)$. So Lemma $3.4($ iii) holds.

The proof of Lemma 3.4 is now complete. 
Lemma 3.5. Suppose that $f \in C^{2}[0, \infty)$ satisfies (H1) and (H3)-(H5). Then, for $H_{c}(\rho, q(\rho, c))$ with $\rho \geq \rho_{0}$ and $c>0$, the following assertions (i)-(iii) hold:

(i) There exists a unique positive $c_{1}$ such that

$$
\lim _{\rho \rightarrow \rho_{0}(c)^{+}} \frac{d}{d \rho} H_{c}(\rho, q(\rho, c)) \begin{cases}>0 & \text { when } c \in\left(0, c_{1}\right), \\ =0 & \text { when } c=c_{1}, \\ <0 & \text { when } c \in\left(c_{1}, \infty\right) .\end{cases}
$$

(ii) If $f$ satisfies $(\mathrm{H} 6)$, then for $c=c_{1}$, there exists $\widetilde{\rho}>\rho_{0}\left(c_{1}\right)$ such that $\frac{d}{d \rho} H_{c_{1}}\left(\rho, q\left(\rho, c_{1}\right)\right)$ $<0$ for $\rho_{0}\left(c_{1}\right)<\rho<\widetilde{\rho}$.

(iii) $\frac{d}{d \rho} H_{c}(\rho, q(\rho, c))>0$ for $0<c<c_{1}$ and $\rho_{0}(c)<\rho<\rho_{0}\left(c_{1}\right)$.

Proof. Lemma 3.5(iii) is from [11, Lemma 3.5(iii)]. We then prove Lemma 3.5(i) and (ii) as follows.

(i) Notice that, by 3.15$)$, studying the sign of $\lim _{\rho \rightarrow \rho_{0}(c)^{+}} \frac{d}{d \rho} H_{c}(\rho, q(\rho, c))$ is equivalent to studying that of $\Phi(\rho)$. Then we have that, for $\rho>0$,

$$
\begin{aligned}
\Phi(\rho)= & \sqrt{2} G^{\prime}(\rho) f(0)-\frac{f(\rho)}{\sqrt{F(\rho)}} \quad(\text { by }(3.16)) \\
= & \int_{0}^{\rho} \frac{f(0)[\theta(\rho)-\theta(s)]}{\rho[F(\rho)-F(s)]^{3 / 2}} d s-\frac{f(\rho)}{\sqrt{F(\rho)}} \int_{0}^{\rho} \frac{d s}{2 \sqrt{\rho(\rho-s)}} \\
& \quad\left(\text { by }(3.2) \text { and since } \int_{0}^{\rho} \frac{d s}{2 \sqrt{\rho(\rho-s)}}=1\right) \\
= & \int_{0}^{\rho} \frac{R(\rho, s)}{2 \rho[F(\rho)-F(s)]^{3 / 2}} d s,
\end{aligned}
$$

where the function $R(\rho, s)$ is defined in 3.6$)$. Then, by Lemma 3.1(ix)-(x), we have that

$$
\Phi(\rho)<0 \quad \text { for } \rho>\bar{\rho} \text {. }
$$

Next, we show that $\lim _{\rho \rightarrow 0^{+}} \Phi(\rho)=\infty$. Note that, by integration by parts, we have that

$$
\sqrt{2} G^{\prime}(\rho)=2 \frac{f(\rho)}{f(0) \sqrt{F(\rho)}}-2 \int_{0}^{\rho} \frac{f^{\prime}(s) f(\rho)}{[f(s)]^{2} \sqrt{F(\rho)-F(s)}} d s,
$$

see [11, Proof of Lemma 3.6 on p. 8375]. So we can represent, by $3.16, \Phi(\rho)$ as follows:

$$
\Phi(\rho)=\frac{f(\rho)}{\sqrt{F(\rho)}}-2 f(0) \int_{0}^{\rho} \frac{f^{\prime}(s) f(\rho)}{[f(s)]^{2} \sqrt{F(\rho)-F(s)}} d s .
$$

Consequently, let $\breve{\rho}>0$ be an arbitrary number such that, for $0<\rho \leq \breve{\rho}$, $0<f(0)<$ $f(\rho) \leq 2 f(0)$ and $f^{\prime}(\rho) \leq 2 f^{\prime}(0)$, where the existence of $\breve{\rho}>0$ follows directly by (H1). 
Then, for $0<\rho \leq \breve{\rho}$,

$$
\begin{aligned}
\Phi(\rho) & \geq \frac{f(\rho)}{\sqrt{F(\rho)}}-2 f(0) \int_{0}^{\rho} \frac{4 f^{\prime}(0) f(0)}{[f(0)]^{2} \sqrt{F(\rho)-F(s)}} d s \\
& \geq \frac{f(\rho)}{\sqrt{F(\rho)}}-8 f^{\prime}(0) \int_{0}^{\rho} \frac{1}{\sqrt{F(\rho)-F(s)}} d s \\
& =\frac{f(\rho)}{\sqrt{F(\rho)}}-4 \sqrt{2} f^{\prime}(0) G(\rho) .
\end{aligned}
$$

Hence $\lim _{\rho \rightarrow 0^{+}} \Phi(\rho)=\infty$ since $\lim _{\rho \rightarrow 0^{+}} G(\rho)=0$ by (3.4), $F(0)=0$ and $f(0)>0$.

Finally, for $0<\rho<\tau$, we compute that

$$
\begin{aligned}
& \Phi^{\prime}(\rho)+\frac{M_{2}(\rho)+1}{2 \rho} \Phi(\rho) \\
= & \sqrt{2} f(0)\left[G^{\prime \prime}(\rho)+\frac{M_{2}(\rho)+1}{2 \rho} G^{\prime}(\rho)\right]-\left\{\left[\frac{f(\rho)}{\sqrt{F(\rho)}}\right]^{\prime}+\frac{M_{2}(\rho)+1}{2 \rho} \frac{f(\rho)}{\sqrt{F(\rho)}}\right. \\
< & \sqrt{2} f(0)\left[G^{\prime \prime}(\rho)+\frac{M_{2}(\rho)+1}{2 \rho} G^{\prime}(\rho)\right] \quad(\text { by Lemma 3.1 (viii)) } \\
= & f(0) \int_{0}^{\rho} \frac{\frac{3}{2}\left[P_{1}(\rho, s)\right]^{2}-\left[P_{2}(\rho, s)+2+\frac{M_{2}(\rho)+1}{2}\right] P_{1}(\rho, s)+\left[M_{2}(\rho)+1\right]}{\rho^{2} \sqrt{F(\rho)-F(s)}} d s
\end{aligned}
$$

(by 3.2 and $(3.3)$

$$
\begin{aligned}
& <f(0) \int_{0}^{\rho} \frac{\frac{3}{2}\left[P_{1}(\rho, s)\right]^{2}-\frac{3}{2}\left[M_{2}(\rho)+\frac{5}{3}\right] P_{1}(\rho, s)+\left[M_{2}(\rho)+1\right]}{\rho^{2} \sqrt{F(\rho)-F(s)}} d s \quad \text { (by Lemma 3.1(vi)) } \\
& =f(0) \int_{0}^{\rho} \frac{\frac{3}{2}\left[P_{1}(\rho, s)-\frac{2}{3}\right]\left\{P_{1}(\rho, s)-\left[M_{2}(\rho)+1\right]\right\}}{\rho^{2} \sqrt{F(\rho)-F(s)}} d s<0
\end{aligned}
$$

by Lemma 3.1(iv)-(v), which implies that $\Phi^{\prime}(\rho)<0$ whenever $\Phi(\rho)=0$ on $(0, \tau)$. Combining the facts that $\lim _{\rho \rightarrow 0^{+}} \Phi(\rho)=\infty$ and $\Phi(\bar{\rho}) \leq 0$ by 3.19 , we conclude that there exists a unique $\rho_{0}^{*}$ on $(0, \bar{\rho}]$ such that

$$
\Phi(\rho) \begin{cases}>0 & \text { when } \rho \in\left(0, \rho_{0}^{*}\right), \\ =0 & \text { when } \rho=\rho_{0}^{*}, \\ <0 & \text { when } \rho \in\left(\rho_{0}^{*}, \infty\right) .\end{cases}
$$

By Lemma 3.2(vii), there exists $c_{1}>0$ such that $\rho_{0}\left(c_{1}\right)=\rho_{0}^{*}$. Moreover, $\rho_{0}(c) \in\left(0, \rho_{0}^{*}\right)$ (resp., $\left.\left(\rho_{0}^{*}, \infty\right)\right)$ if and only if $c \in\left(0, c_{1}\right)$ (resp., $c \in\left(c_{1}, \infty\right)$ ). Hence we have that

$$
\Phi\left(\rho_{0}(c)\right) \begin{cases}>0 & \text { when } c \in\left(0, c_{1}\right), \\ =0 & \text { when } c=c_{1}, \\ <0 & \text { when } c \in\left(c_{1}, \infty\right) .\end{cases}
$$


Then, by 3.15), Lemma 3.5(i) holds.

(ii) Let $\rho_{0}^{*}\left(=\rho_{0}\left(c_{1}\right)\right)$ be defined in 3.20$)$. Then $\Psi\left(\rho_{0}^{*}, q\left(\rho_{0}^{*}, c_{1}\right)\right)=\Psi\left(\rho_{0}^{*}, 0\right)=$ $\frac{2}{f(0)} \Phi\left(\rho_{0}^{*}\right)=0$ by Lemma 3.2(iv), 3.14, 3.16) and 3.20. So if we can show that

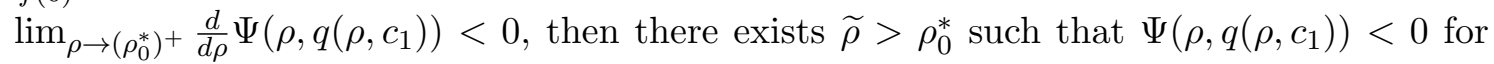
$\rho_{0}^{*}<\rho<\widetilde{\rho}$, and hence $\frac{d}{d \rho} H_{c_{1}}\left(\rho, q\left(\rho, c_{1}\right)\right)<0$ for $\rho_{0}^{*}<\rho<\widetilde{\rho}$ by (3.13), which completes the proof. Indeed, we compute that, by (3.8) and (3.14),

$$
\begin{aligned}
\frac{d}{d \rho} \Psi(\rho, q(\rho, c))= & 2 \sqrt{2} G^{\prime \prime}(\rho)-2 \frac{f^{\prime}(\rho) f(q(\rho, c))-f^{\prime}(q(\rho, c)) \frac{\partial}{\partial \rho} q(\rho, c) f(\rho)}{[f(q(\rho, c))]^{2} \sqrt{F(\rho)-F(q(\rho, c))}} \\
& +\frac{[f(\rho)]^{2}}{f(q(\rho, c))[F(\rho)-F(q(\rho, c))]^{3 / 2}} \\
& +\int_{0}^{q(\rho, c)} \frac{f^{\prime}(\rho)[F(\rho)-F(s)]-\frac{3}{2}[f(\rho)]^{2}}{[F(\rho)-F(s)]^{5 / 2}} d s .
\end{aligned}
$$

It follows that

$$
\begin{aligned}
& \lim _{\rho \rightarrow\left(\rho_{0}^{*}\right)^{+}} \frac{d}{d \rho} \Psi\left(\rho, q\left(\rho, c_{1}\right)\right) \\
& =2 \sqrt{2} G^{\prime \prime}\left(\rho_{0}^{*}\right)+\frac{-2\left\{f(0) f^{\prime}\left(\rho_{0}^{*}\right)-\frac{f^{\prime}(0)}{f(0)}\left[f\left(\rho_{0}^{*}\right)\right]^{2}\right\} F\left(\rho_{0}^{*}\right)+f(0)\left[f\left(\rho_{0}^{*}\right)\right]^{2}}{[f(0)]^{2}\left[F\left(\rho_{0}^{*}\right)\right]^{3 / 2}} \\
& \text { (since } q\left(\rho_{0}^{*}, c_{1}\right)=0 \text { and } \lim _{\rho \rightarrow\left(\rho_{0}^{*}\right)+} \frac{\partial}{\partial \rho} q\left(\rho, c_{1}\right)=\frac{f\left(\rho_{0}^{*}\right)}{f(0)} \text { by 3.12) and 3.17) } \\
& =2 \sqrt{2} G^{\prime \prime}\left(\rho_{0}^{*}\right)+\frac{N_{1}\left(\rho_{0}^{*}\right)}{\rho_{0}^{*}} \frac{f\left(\rho_{0}^{*}\right)}{f(0) \sqrt{F\left(\rho_{0}^{*}\right)}}\left(N_{1}(\rho) \text { is defined in 1.5 }\right) \\
& =2 \sqrt{2} G^{\prime \prime}\left(\rho_{0}^{*}\right)+\frac{N_{1}\left(\rho_{0}^{*}\right)}{\rho_{0}^{*}}\left[\sqrt{2} G^{\prime}\left(\rho_{0}^{*}\right)\right] \quad(\text { by } 3.18 \text { and } 3.20) \\
& =\int_{0}^{\rho_{0}^{*}} \frac{3\left[P_{1}\left(\rho_{0}^{*}, s\right)\right]^{2}-2\left[P_{2}\left(\rho_{0}^{*}, s\right)+2\right] P_{1}\left(\rho_{0}^{*}, s\right)+N_{1}\left(\rho_{0}^{*}\right)\left[2-P_{1}\left(\rho_{0}^{*}, s\right)\right]}{\rho_{0}^{* 2} \sqrt{F\left(\rho_{0}^{*}\right)-F(s)}} d s \\
& =\int_{0}^{\rho_{0}^{*}} \frac{3\left[P_{1}\left(\rho_{0}^{*}, s\right)-1\right]\left\{P_{1}\left(\rho_{0}^{*}, s\right)-\left[M_{2}\left(\rho_{0}^{*}\right)+1\right]\right\}-P_{1}\left(\rho_{0}^{*}, s\right)\left[N_{2}\left(\rho_{0}^{*}\right)+2 P_{2}\left(\rho_{0}^{*}, s\right)\right]-N_{3}\left(\rho_{0}^{*}\right)}{\rho_{0}^{* 2} \sqrt{F\left(\rho_{0}^{*}\right)-F(s)}} d s \\
& \left(N_{2}(\rho) \text { and } N_{3}(\rho) \text { are defined in }(1.6)\right) \\
& \leq \int_{0}^{\rho_{0}^{*}} \frac{-P_{1}\left(\rho_{0}^{*}, s\right)\left[N_{2}\left(\rho_{0}^{*}\right)+2 P_{2}\left(\rho_{0}^{*}, s\right)\right]-N_{3}\left(\rho_{0}^{*}\right)}{\rho_{0}^{* 2} \sqrt{F\left(\rho_{0}^{*}\right)-F(s)}} d s \quad \text { (by Lemma 3.1.iv) and (v)) } \\
& =-\int_{0}^{\rho_{0}^{*}} \frac{W\left(\rho_{0}^{*}, s\right)+N_{3}\left(\rho_{0}^{*}\right)}{\rho_{0}^{* 2} \sqrt{F\left(\rho_{0}^{*}\right)-F(s)}} d s,
\end{aligned}
$$

where $W(\rho, s)$ is defined in 1.8$)$. Then to prove $\lim _{\rho \rightarrow\left(\rho_{0}^{*}\right)^{+}} \frac{d}{d \rho} \Psi\left(\rho, q\left(\rho, c_{1}\right)\right)<0$, it suffices to prove that

$$
\int_{0}^{\rho} \frac{W(\rho, s)+N_{3}(\rho)}{\sqrt{F(\rho)-F(s)}} d s>0 \quad \text { for } 0<\rho \leq \bar{\rho}
$$

since $\rho_{0}^{*} \leq \bar{\rho}$. We shall prove 3.21 for each $\rho \in(0, \bar{\rho}]$ in the following two cases. 
Case 1: $W(\rho, 0)+N_{3}(\rho) \geq 0$ for $\rho \in(0, \bar{\rho}]$. We have that $W(\rho, s)+N_{3}(\rho) \geq W(\rho, 0)+$ $s \widetilde{W}_{0}(\rho)+N_{3}(\rho)>0$ for $s \in(0, \rho]$ by 1.11$)$ and 1.12$)$ in (H6). Hence (3.21) holds.

Case 2: $W(\rho, 0)+N_{3}(\rho)<0$ for $\rho \in(0, \bar{\rho}]$. We have that

$$
\begin{aligned}
& \int_{0}^{\rho} \frac{W(\rho, s)+N_{3}(\rho)}{\sqrt{F(\rho)-F(s)}} d s \\
\geq & \left.\int_{0}^{\rho} \frac{W(\rho, 0)+s \widetilde{W}_{0}(\rho)+N_{3}(\rho)}{\sqrt{F(\rho)-F(s)}} d s \quad \text { (by }(1.12) \text { in }(\mathrm{H} 6)\right) \\
= & \int_{0}^{\rho} \frac{W(\rho, 0)+N_{3}(\rho)}{\sqrt{F(\rho)-F(s)}} d s+\int_{0}^{\rho} \frac{s \widetilde{W}_{0}(\rho)}{\sqrt{F(\rho)-F(s)}} d s \\
\geq & \int_{0}^{\rho} \frac{W(\rho, 0)+N_{3}(\rho)}{\sqrt{f(0)} \sqrt{\rho-s}} d s+\int_{0}^{\rho} \frac{s \widetilde{W}_{0}(\rho)}{\sqrt{f(\rho)} \sqrt{\rho-s}} d s \\
= & \frac{2 \sqrt{ } \operatorname{by}}{3 \sqrt{f(11)},(\text { H1) and the Mean Value Theorem) } \sqrt{f(\rho)}}\left\{3 \sqrt{f(\rho)}\left[W(\rho, 0)+N_{3}(\rho)\right]+2 \rho \sqrt{f(0) W_{0}}(\rho)\right\}>0
\end{aligned}
$$

by 1.13 in (H6).

By Cases 1 and 2, (3.21) holds. So Lemma 3.5(ii) holds.

The proof of Lemma 3.5 is now complete.

The next lemma is from [11, Lemma 3.6].

Lemma 3.6. Suppose that $f \in C^{2}[0, \infty)$ satisfies (H1) and (H3)-(H5). Then, for $G(\rho)$ with $\rho>0$ and for $H_{c}(\rho, q(\rho, c))$ with $\rho \geq \rho_{0}$ and $c>0$, if $G^{\prime}(\rho) \leq 0$ for some $\rho>0$, then $\frac{d}{d \rho} H_{c}(\rho, q(\rho, c))<0$ for $0<c \leq \widehat{c}$. Here $\widehat{c}=\sqrt{2 F(\rho)} G(\rho)$ is defined in Lemma 3.2(viii).

In the next Lemma 3.7, we show some properties of the nonlinearity $f(\rho)(=f(\rho, a))=$ $\exp \left(\frac{a \rho}{a+\rho}\right)$ with $a \geq 4$. When there is no confusion arising, throughout the paper, we would omit the parameter $a$ in representing functions resulting from $f(\rho)(=f(\rho, a))=\exp \left(\frac{a \rho}{a+\rho}\right)$ for the reason of clarity such as the functions defined in $(1.2)-(1.9)$.

Lemma 3.7. Consider $f(\rho)=\exp \left(\frac{a \rho}{a+\rho}\right)$ with $a \geq 4.37$, and let $N_{2}(\rho)\left(=N_{2}(\rho, a)\right)$ be defined in (1.6). Then the following assertions (i)-(iii) hold:

(i) There exists a unique positive zero $\bar{\rho}$ of $2 f(0)\left[2-M_{1}(\rho)\right]-f(\rho)$ and $\bar{\rho}<1 / 2$.

(ii) For $0<\rho \leq \bar{\rho}, N_{2}(\rho, a)$ is a strictly decreasing function of a on $[4.37, \infty)$ and $-2<N_{2}(\rho)<-1$.

(iii) For $0<\rho \leq \bar{\rho}, N_{4}(\rho)\left(=N_{4}(\rho, a)\right) \equiv F(\rho) \widetilde{W}_{0}(\rho)$ is a positive, strictly increasing function of a on $[4.37, \infty)$.

The proof of Lemma 3.7 is lengthy, and hence it is given in 9 . 


\section{Proofs of the main results}

In this section, we prove our main results (Theorems 2.1, 2.3 2.5). We note that, from the relationship between bifurcation curve $S$ and time map $G$ as in (3.1), and that between bifurcation curves $\widetilde{S}_{c}$ and the time map $H_{c}$ as in (3.11), the assertions in Theorems 2.1. 2.3 and 2.4 can be concluded directly from Lemmas 3.1 3.6. Hence we shall just give the main framework of these proofs; cf. proofs of [11, Theorems 2.1-2.3] in [11, Section 4].

Proof of Theorem 2.1. First, by 3.10, 3.11) and Lemma 3.2(iv), for any $c>0$, the bifurcation curve $\widetilde{S}_{c}$ is a continuous curve which starts at some point $\left(\lambda_{0},\left\|u_{\lambda_{0}}\right\|_{\infty}\right)$ on the $\left(\lambda,\left\|u_{\lambda}\right\|_{\infty}\right)$-plane with $\lambda_{0}\left(=H\left(\rho_{0}(c), q\left(\rho_{0}(c), c\right)\right)\right)=H\left(\rho_{0}(c), 0\right)>0$ and $\left\|u_{\lambda}\right\|_{\infty}=\rho_{0}>0$. In addition, Theorem 2.1(i) and (iii) follow from Lemma 3.3(i), while Theorem 2.1(ii) and (iv) follow from Lemma 3.3 (ii) and Lemma 3.2(vii), respectively. Hence the proof of Theorem 2.1 is complete.

Proof of Theorem 2.3. Theorem 2.3(i) and (ii) follow from Lemma 3.4(i) and (ii), respectively.

Proof of Theorem 2.4. We first note that, from the relationship between the bifurcation curve $S$ and time map $G$ as in (3.1) and since the bifurcation curve $S$ of (1.14) is exactly type $1 S$-shaped on the $\left(\lambda,\left\|u_{\lambda}\right\|_{\infty}\right)$-plane by Theorem 1.2 , there exist two positive $\bar{\rho}_{1}<\bar{\rho}_{2}$ such that

$$
G^{\prime}(\rho) \begin{cases}\geq 0 & \text { when } \rho \in\left(0, \bar{\rho}_{1}\right) \cup\left(\bar{\rho}_{2}, \infty\right), \\ =0 & \text { when } \rho=\bar{\rho}_{1} \text { or } \bar{\rho}_{2}, \\ \leq 0 & \text { when } \rho \in\left(\bar{\rho}_{1}, \bar{\rho}_{2}\right) .\end{cases}
$$

Let $c_{1}$ and $\rho_{0}^{*}\left(=\rho_{0}\left(c_{1}\right)\right)$ be defined as in 3.17 and 3.20 , respectively. Then we have that

$$
\rho_{0}^{*}\left(=\rho_{0}\left(c_{1}\right)\right)<\bar{\rho}_{1}
$$

since $H_{c}(\rho, q(\rho, c)) \leq[G(\rho)]^{2}$ for any $c>0$ and $\rho \geq \rho_{0}$ by Lemma 3.3(i) and since

$$
\lim _{\rho \rightarrow \rho_{0}(c)^{+}} \frac{d}{d \rho} H_{c_{1}}(\rho, q(\rho, c)) \geq 0
$$

for $c \leq c_{1}$ by 3.17 .

(I) We prove Theorem 2.4(i). Let $0<c<c_{1}$. Then $H_{c}(\rho, q(\rho, c))$ is defined on the interval $\left[\rho_{0}(c), \infty\right)$ with $0<\rho_{0}(c)<\rho_{0}^{*}<\bar{\rho}_{1}$ by Lemma 3.2(vii) and 4.1). Consequently, since (a) $\lim _{\rho \rightarrow \rho_{0}(c)^{+}} \frac{d}{d \rho} H_{c}(\rho, q(\rho, c))>0$ by Lemma $3.5(\mathrm{i})$, (b) $\frac{d}{d \rho} H_{c}(\rho, q(\rho, c))<0$ for $\bar{\rho}_{1} \leq \rho \leq \bar{\rho}_{2}$ by Lemma 3.6, and (c) $\lim _{\rho \rightarrow \infty} H_{c}(\rho, q(\rho, c))=\infty$ by Lemma 3.3(ii), we conclude that the bifurcation curve $\widetilde{S}_{c}$ is $S$-shaped on the $\left(\lambda,\|u\|_{\infty}\right)$-plane. We next show 
that the $S$-shaped bifurcation curve $\widetilde{S}_{c}$ can be of either type 1 , type 2 or type 3 for each different value of $c$ on $\left(0, c_{1}\right)$. In fact, since $\lim _{c \rightarrow 0^{+}} \lambda_{\min }(c)=0$ by Lemma 3.4(ii) and since $H_{c}(\rho, q(\rho, c))>\frac{1}{4}[G(\rho)]^{2}$ by Lemma 3.3(iii), there exists $c_{1,1} \in\left(0, c_{1}\right)$ such that, for $0<c<c_{1,1}$, the $S$-shaped bifurcation curve $\widetilde{S}_{c}$ is of type 1 on the $\left(\lambda,\|u\|_{\infty}\right)$ plane. In addition, since $(\mathrm{d})$ there exists $\widetilde{\rho}>\rho_{0}\left(c_{1}\right)$ such that $\frac{d}{d \rho} H_{c_{1}}\left(\rho, q\left(\rho, c_{1}\right)\right)<0$ for $\rho_{0}\left(c_{1}\right)<\rho<\widetilde{\rho}$ by Lemma 3.5(ii), (e) $H_{c}(\rho, q(\rho, c))<H_{c_{1}}\left(\rho, q\left(\rho, c_{1}\right)\right)$ for $0<c<c_{1}$ by Lemma 3.4 (i), and (f) $\lim _{\rho \rightarrow \rho_{0}(c)}+\frac{d}{d \rho} H_{c}(\rho, q(\rho, c))>0$ for $0<c<c_{1}$ by Lemma 3.5(i), there exists $c_{1,3} \in\left[c_{1,1}, c_{1}\right)$ such that, for $c_{1,3}<c<c_{1}$, the $S$-shaped bifurcation curve $\widetilde{S}_{c}$ is of type 3 on the $\left(\lambda,\|u\|_{\infty}\right)$-plane. Moreover, by the continuity of evolution of bifurcation curves $\widetilde{S}_{c}$ from $0^{+}$to $c_{1}^{-}$, there exists $c_{1,2} \in\left[c_{1,1}, c_{1,3}\right]$ such that the $S$-shaped bifurcation curve $\widetilde{S}_{c_{1,2}}$ is of type 2 on the $\left(\lambda,\|u\|_{\infty}\right)$-plane.

(II) We prove Theorem 2.4(ii). Let $c \geq c_{1}$. Since $\frac{d}{d \rho} H_{c}(\rho, q(\rho, c))<0$ for $\rho$ is sufficient close to $\left(\rho_{0}\right)^{+}$by Lemma 3.5 (i)-(ii) and since $\lim _{\rho \rightarrow \infty} H_{c}(\rho, q(\rho, c))=\infty$, we have that the bifurcation curve $\widetilde{S}_{c}$ is $\subset$-shaped on the $\left(\lambda,\|u\|_{\infty}\right)$-plane for $c \geq c_{1}$.

Finally, the multiplicity results of positive solutions for 1.1 with $c>0$ follow immediately from the shape of bifurcation curve $\widetilde{S}_{c}$. Hence the proof of Theorem 2.4 is now complete.

Proof of Theorem 2.5. Let $f(\rho)(=f(\rho, a))=\exp \left(\frac{a \rho}{a+\rho}\right)$. Then, for $a>0,(\mathrm{H} 1)$ and (H3) hold as provided in [6, Theorem 2.2(i)]. While, for $a \geq 4.37$, (H2) holds with $\gamma=a(a-2) / 2>0$ as provided in $[6$, Theorem 2.2(i)]. We then verify hypotheses (H4)(H5) for $a>0$ and (H6) for $a \geq 4.37$ as follows.

(I) We verify (H4) for $a>0$ with choosing

$$
\tau=a \text {. }
$$

We compute that

$$
\left[f^{\prime}(u)+u f^{\prime \prime}(u)\right] f(u)-u\left[f^{\prime}(u)\right]^{2}=\frac{a^{2}(a-u)}{(a+u)^{3}} \exp \left(\frac{2 a u}{a+u}\right) \begin{cases}>0 & \text { when } u \in[0, a), \\ =0 & \text { when } u=\tau=a, \\ <0 & \text { when } u \in(a, \infty) .\end{cases}
$$

So (H4) holds for $a>0$ with $\tau=a$.

(II) We verify (H5) for $a>0$. By direct computation, we have that

$$
\left[f^{\prime}(u)\right]^{2}-f^{\prime \prime}(u) f(u)=\frac{2 a^{2}}{(a+u)^{3}} \exp \left(\frac{2 a u}{a+u}\right)>0
$$

for $u \geq 0$. So (H5) holds for $a>0$.

(III) We verify (H6) for $a \geq 4.37$. It suffices to prove $1.10-1.13$ for $a \geq 4.37$. We first note that the assertions given in Lemma 3.1(i)-(viii) hold since (H1) and (H3)-(H5) hold for $a>0$ as claimed above. 
(i) We verify 1.10 for $a \geq 4.37$. We compute that

$$
f(\tau)-4 f(0)=\exp \left(\frac{a}{2}\right)-4 \geq \exp \left(\frac{4.37}{2}\right)-4>\exp (2)-4>0 .
$$

So 1.10 holds for $a \geq 4.37$.

Then, by Lemma 3.1(ix), Lemma 3.7(i) and 4.2), we have that $0<\bar{\rho}(<1 / 2)<\tau(=$ a) for $a \geq 4.37$.

(ii) We verify 1.11 for $a \geq 4.37$. We compute that, for $0<\rho<\bar{\rho}(<1 / 2)$,

$$
\begin{aligned}
\widetilde{W}_{0}(\rho) & =\frac{f(0)}{F(\rho)}\left\{\left[M_{1}(\rho)-1\right] N_{2}(\rho)+2 M_{1}(\rho) M_{2}(\rho)\right\} \\
& \geq \frac{f(0)}{F(\rho)}\left\{-2\left[M_{1}(\rho)-1\right]+2 M_{1}(\rho) M_{2}(\rho)\right\}
\end{aligned}
$$

(since $M_{1}(\rho)=P_{1}(\rho, 0)>1$ by Lemma 3.1(iv) and $N_{2}(\rho)>-2$ by Lemma 3.7(ii))

$$
=\frac{2 f(0)}{F(\rho)}\left\{\left[M_{2}(\rho)-1\right] M_{1}(\rho)+1\right\} \text {. }
$$

Moreover, for $0<\rho<\bar{\rho}(<1 / 2)$, since $M_{2}(\rho)-1<M_{2}(1 / 2)-1=-\left(2 a^{2}+4 a+1\right) /(2 a+$ $1)^{2}<0$ by the fact that $M_{2}(\rho)$ is a strictly increasing function of $\rho$ on $[0, a]$ as claimed in Lemma 3.1(ii) and since $M_{1}(\rho)<M_{2}(\rho)+1$ by Lemma 3.1(v), we conclude that

$$
\widetilde{W}_{0}(\rho) \geq \frac{2 f(0)}{F(\rho)}\left\{\left[M_{2}(\rho)-1\right]\left[M_{2}(\rho)+1\right]+1\right\}=\frac{2 f(0)}{F(\rho)}\left[M_{2}(\rho)\right]^{2}>0 .
$$

So (1.11) holds $a \geq 4.37$.

(iii) We verify 1.12 for $a \geq 4.37$. Define $W_{1}(\rho, s)=W(\rho, s)-\left[W(\rho, 0)+s \widetilde{W}_{0}(\rho)\right]$ for $0 \leq s<\rho$. Then showing 1.12 is equivalent to showing that $W_{1}(\rho, s) \geq 0$ for $0 \leq s<\rho \leq \bar{\rho}$. It is easy to see that $W_{1}(\rho, 0)=0$ and $\left[\frac{\partial}{\partial s} W_{1}(\rho, s)\right]_{s=0}=0$. Moreover, by direct computation, we have that

$$
\begin{aligned}
\frac{\partial}{\partial s} W_{1}(\rho, s) & =\frac{\partial}{\partial s} W(\rho, s)-\widetilde{W}_{0}(\rho)=\frac{f(s)}{F(\rho)-F(s)}[W(\rho, s)-U(\rho, s)]-\widetilde{W}_{0}(\rho), \\
\frac{\partial^{2}}{\partial s^{2}} W_{1}(\rho, s) & =\frac{f(s)}{F(\rho)-F(s)}\left[V(\rho, s) \frac{\partial}{\partial s} W(\rho, s)-\frac{\partial}{\partial s} U(\rho, s)\right],
\end{aligned}
$$

where

$$
U(\rho, s) \equiv\left[1+\frac{s f^{\prime}(s)}{f(s)}\right] N_{2}(\rho)+\frac{4 s f^{\prime}(s)+2 s^{2} f^{\prime \prime}(s)}{f(s)}, \quad V(\rho, s) \equiv \frac{f^{\prime}(s)}{[f(s)]^{2}}[F(\rho)-F(s)]+2 .
$$

Hence, for any fixed $\rho \in(0, \bar{\rho}]$, if $0 \leq s<\rho$ satisfies $\frac{\partial}{\partial s} W_{1}(\rho, s)=0$, then

$$
\frac{\partial^{2}}{\partial s^{2}} W_{1}(\rho, s)=\frac{f(s)}{F(\rho)-F(s)} W_{2}(\rho, s)
$$

where

$$
W_{2}(\rho, s) \equiv V(\rho, s) \widetilde{W}_{0}(\rho)-\frac{\partial}{\partial s} U(\rho, s)
$$


Applying the relationship between $W_{1}(\rho, s)$ and $W_{2}(\rho, s)$ in 4.3, we claim that, for any fixed $\rho \in(0, \bar{\rho}], W_{1}(\rho, s)$ is an increasing function of $s$ on $[0, \rho)$ if the following assertions (a)-(d) hold:

(a) $\frac{\partial^{2}}{\partial s^{2}} W_{2}(\rho, s)>0$ for $0 \leq s<\rho \leq \bar{\rho}(<1 / 2)$.

(b) $W_{2}(\rho, 0)>0$ for $0<\rho \leq \bar{\rho}(<1 / 2)$.

(c) $\lim _{s \rightarrow \rho^{-}} W_{2}(\rho, s)<0$ for $0<\rho \leq \bar{\rho}(<1 / 2)$.

(d) $\lim _{s \rightarrow \rho^{-}} W_{2}(\rho, s)=-2 \lim _{s \rightarrow \rho^{-}}\left[\frac{\partial}{\partial s} W_{1}(\rho, s)\right]$ for $0<\rho \leq \bar{\rho}(<1 / 2)$.

Indeed, suppose that $W_{1}(\rho, s)$ is not an increasing function of $s$ on $[0, \rho)$ for some fixed $\rho \in(0, \bar{\rho}]$. Then there exists some $\bar{s} \in(0, \rho)$ such that $\frac{\partial}{\partial s} W_{1}(\rho, \bar{s})<0$. It follows that there exists some $s_{*} \in(0, \bar{s})$, which is a local maximum of $W_{1}(\rho, s)$ on $(0, \bar{s})$ since $\left[\frac{\partial}{\partial s} W_{1}(\rho, s)\right]_{s=0}=0$ and $\left[\frac{\partial^{2}}{\partial s^{2}} W_{1}(\rho, s)\right]_{s=0}>0$ by (4.3) and assertion (b). So $\frac{\partial}{\partial s} W_{1}\left(\rho, s_{*}\right)=$ 0 and $\frac{\partial^{2}}{\partial s^{2}} W_{1}\left(\rho, s_{*}\right) \leq 0$. Following by 4.3$)$, we have that $W_{2}\left(\rho, s_{*}\right) \leq 0$. Consequently, $W_{2}(\rho, s)<0$ for $s_{*}<s<\rho$ by assertions (a)-(c). However, since $\frac{\partial}{\partial s} W_{1}(\rho, \bar{s})<0$ and $\lim _{s \rightarrow \rho^{-}}\left[\frac{\partial}{\partial s} W_{1}(\rho, s)\right]>0$ by assertions (c)-(d), there exists some $s^{*} \in(\bar{s}, \rho)$, which is a local minimum of $W_{1}(\rho, s)$ on $(\bar{s}, \rho)$. So $\frac{\partial}{\partial s} W_{1}\left(\rho, s^{*}\right)=0$ and $\frac{\partial^{2}}{\partial s^{2}} W_{1}\left(\rho, s^{*}\right) \geq 0$. Again, by (4.3), $W_{2}\left(\rho, s^{*}\right) \geq 0$. So we get a contradiction to the fact that $W_{2}(\rho, s)<0$ for $s_{*}<s<\rho$, and hence $W_{1}(\rho, s)$ is an increasing function of $s$ on $[0, \rho)$. Thus $W_{1}(\rho, s) \geq W_{1}(\rho, 0)=0$ for $0 \leq s<\rho \leq \bar{\rho}$. So 1.12 holds for $a \geq 4.37$.

The proofs of assertions (a)-(d) for $f(\rho)=\exp \left(\frac{a \rho}{a+\rho}\right)$ with $a \geq 4.37$ are lengthy, and hence they are given in 9$]$.

(iv) We verify 1.13) for $a \geq 4.37$. We compute that

$$
\begin{aligned}
& 3 \sqrt{f(\rho)}\left[W(\rho, 0)+N_{3}(\rho)\right]+2 \rho \sqrt{f(0)} \widetilde{W}_{0}(\rho) \\
= & F(\rho) \widetilde{W}_{0}(\rho)\left[3 \frac{\sqrt{f(\rho)}}{f(0)}+\frac{2 \sqrt{f(0)} \rho}{F(\rho)}\right] \\
& +3 \sqrt{f(\rho)}\left[1-2 \frac{f^{\prime}(0)}{[f(0)]^{2}} \rho f(\rho)-M_{1}(\rho)+2 M_{2}(\rho)\right] \\
= & N_{4}(\rho, a)\left[3 \sqrt{f(\rho)}+\frac{2 \rho}{F(\rho)}\right]+3 \sqrt{f(\rho)}\left[1-N_{1}(\rho, a)\right] \quad\left(\text { since } f(0)=f^{\prime}(0)=1\right) \\
\equiv & N_{4}(\rho, a) N_{5}(\rho, a)+N_{6}(\rho, a) \equiv N_{7}(\rho, a) .
\end{aligned}
$$

Here $N_{1}(\rho, a)$ and $N_{4}(\rho, a)$ are defined in 1.5) and Lemma 3.7(iii), respectively. Now, we show that

$$
N_{5}(\rho, a)=3 \sqrt{f(\rho)}+\frac{2 \rho}{F(\rho)} \quad \text { and } \quad N_{6}(\rho, a)=3 \sqrt{f(\rho)}\left[1-N_{1}(\rho, a)\right]
$$

are both strictly increasing functions of $a$ on $[4, \infty)$ for any fixed $\rho \in(0, \bar{\rho}]$. 
We first show that $N_{5}(\rho, a)$ a strictly increasing function of $a$ on $[4, \infty)$ for any fixed $\rho \in(0, \bar{\rho}]$. Let $a \geq 4$ and $0<\rho \leq \bar{\rho}(<1 / 2)$. We compute that

$$
\begin{aligned}
\frac{\partial}{\partial a} N_{5}(\rho, a)= & \frac{2 \rho}{[F(\rho)]^{2}}\left[\frac{3}{4} \frac{\rho[F(\rho)]^{2}}{(a+\rho)^{2}} \exp \left(\frac{a \rho}{2(a+\rho)}\right)-\int_{0}^{\rho} \frac{s^{2}}{(a+s)^{2}} \exp \left(\frac{a s}{a+s}\right) d s\right] \\
\geq & \frac{2 \rho}{[F(\rho)]^{2}}\left[\frac{3}{4} \frac{\rho^{3}}{(a+\rho)^{2}} \exp \left(\frac{a \rho}{2(a+\rho)}\right)-\int_{0}^{\rho} \frac{s^{2}}{(a+s)^{2}} \exp \left(\frac{a s}{a+s}\right) d s\right] \\
& \quad\left(\text { since } F(\rho)=\int_{0}^{\rho} f(s) d s=\int_{0}^{\rho} \exp \left(\frac{a s}{a+s}\right) d s \geq \int_{0}^{\rho} 1 d s=\rho\right) \\
\equiv & \frac{2 \rho}{[F(\rho)]^{2}} N_{8}(\rho, a) .
\end{aligned}
$$

We have that $N_{8}(0, a)=0$ and, for $0<\rho \leq \bar{\rho}(<1 / 2)$,

$$
\begin{aligned}
\frac{\partial}{\partial \rho} N_{8}(\rho, a) & =\frac{\rho^{2} \exp \left(\frac{a \rho}{2(a+\rho)}\right)}{(a+\rho)^{2}}\left[\frac{3}{8} \frac{(\rho+6) a^{2}+8 \rho a+2 \rho^{2}}{(a+\rho)^{2}}-\exp \left(\frac{a \rho}{2(a+\rho)}\right)\right] \\
& \geq \frac{\rho^{2} \exp \left(\frac{a \rho}{2(a+\rho)}\right)}{(a+\rho)^{2}}\left[\frac{3}{8}\left[\frac{(\rho+6) a^{2}+8 \rho a+2 \rho^{2}}{(a+\rho)^{2}}\right]_{a=4}-\exp \left(\frac{\rho}{2}\right)\right] \\
& =\frac{\rho^{2} \exp \left(\frac{a \rho}{2(a+\rho)}\right)}{(a+\rho)^{2}}\left[\frac{3}{8} \frac{2 \rho^{2}+48 \rho+96}{(4+\rho)^{2}}-\exp \left(\frac{\rho}{2}\right)\right] \\
& \geq \frac{\rho^{2} \exp \left(\frac{a \rho}{2(a+\rho)}\right)}{(a+\rho)^{2}}\left[\frac{3}{8} \frac{2 \rho^{2}+48 \rho+96}{(4+\rho)^{2}}-\exp \left(\frac{\rho}{2}\right)\right] \\
& \left(\operatorname{since} \frac{\partial}{\partial \rho}\left[\frac{3}{8} \frac{2 \rho^{2}+4 \rho+2}{(a+\rho)^{2}}>0\right)\right. \\
& \frac{\rho^{2} \exp \left(\frac{a \rho}{2(a+\rho)}\right)}{(a+\rho)^{2}}\left[\frac{241}{108}-\exp \left(\frac{1}{4}\right)\right]>0, \\
&
\end{aligned}
$$

since $241 / 108-\exp (1 / 4)(\approx 0.947)>0$. Hence $N_{8}(\rho, a)>0$ for $a \geq 4$ and $0<\rho \leq \bar{\rho}$. It implies that, by (4.5), $N_{5}(\rho, a)$ is a strictly increasing function of $a$ on $[4, \infty)$ for any fixed $\rho \in(0, \bar{\rho}]$.

We next show that $N_{6}(\rho, a)$ a strictly increasing function of $a$ on $[4, \infty)$ for any fixed $\rho \in(0, \bar{\rho}]$. Let $a \geq 4$ and $0<\rho \leq \bar{\rho}(<1 / 2)$. We compute that

$$
\begin{aligned}
\frac{\partial}{\partial a} N_{6}(\rho, a)= & \frac{3}{2} \frac{\rho^{2}}{(a+\rho)^{2}} \sqrt{f(\rho)}\left[2 M_{2}(\rho)-M_{1}(\rho)-2 \rho f(\rho)+1\right] \\
& +3 \sqrt{f(\rho)}\left[\frac{4 a \rho^{2}}{(a+\rho)^{3}}-\frac{\partial}{\partial a} M_{1}(\rho)-\frac{2 \rho^{3} f(\rho)}{(a+\rho)^{2}}\right]
\end{aligned}
$$


Then, since $M_{1}(\rho)<M_{2}(\rho)+1$ by Lemma 3.1(v) and since

$$
\frac{\partial}{\partial a} M_{1}(\rho, a)=\frac{M_{1}(\rho)}{(a+\rho)^{2}}\left[\rho^{2}-\frac{\frac{\partial}{\partial a} F(\rho)}{F(\rho)}(a+\rho)^{2}\right]<\frac{\rho^{2}}{(a+\rho)^{2}} M_{1}(\rho)<\frac{\rho^{2}}{(a+\rho)^{2}}\left[M_{2}(\rho)+1\right],
$$

we have that

$$
\begin{aligned}
\frac{\partial}{\partial a} N_{6}(\rho, a)> & \frac{3}{2} \frac{\rho^{2}}{(a+\rho)^{2}} \sqrt{f(\rho)}\left\{2 M_{2}(\rho)-\left[M_{2}(\rho)+1\right]-2 \rho f(\rho)+1\right\} \\
& +3 \sqrt{f(\rho)}\left\{\frac{4 a \rho^{2}}{(a+\rho)^{3}}-\left[\frac{\rho^{2}}{(a+\rho)^{2}}\left[M_{2}(\rho)+1\right]\right]-\frac{2 \rho^{3} f(\rho)}{(a+\rho)^{2}}\right\} \\
= & \frac{3 \sqrt{f(\rho)}}{2(a+\rho)^{4}} N_{9}(\rho, a),
\end{aligned}
$$

where

$$
N_{9}(\rho, a) \equiv\left[-6 \rho^{3} f(\rho)-\rho^{3}+6 \rho^{2}\right] a^{2}+\left[-12 \rho^{4} f(\rho)+4 \rho^{3}\right] a-\left[6 \rho^{5} f(\rho)+2 \rho^{4}\right] .
$$

We compute that, for $a \geq 4$ and $0<\rho \leq \bar{\rho}(<1 / 2)$,

$$
\begin{aligned}
\frac{\partial}{\partial a} N_{9}(\rho, a)= & -2 \rho^{2}\left(3 \rho^{3}+6 \rho^{2}+6 a \rho\right) f(\rho)-2 \rho^{2}(-2 \rho+a \rho-6 a) \\
> & -2 \rho^{2}\left(3 \rho^{3}+6 \rho^{2}+6 a \rho\right) \exp (1 / 2)-2 \rho^{2}(-2 \rho+a \rho-6 a) \\
= & 2 a \rho^{2}[-6 \rho \exp (1 / 2)-(\rho-6)]-6 \rho^{2}\left(\rho^{3}+2 \rho^{2}\right) \exp (1 / 2)+4 \rho^{3} \\
\geq & 8 \rho^{2}[-6 \rho \exp (1 / 2)-(\rho-6)]-6 \rho^{2}\left(\rho^{3}+2 \rho^{2}\right) \exp (1 / 2)+4 \rho^{3} \\
& (\operatorname{since}-6 \rho \exp (1 / 2)-(\rho-6)>-3 \exp (1 / 2)-(1 / 2-6)(\approx 0.554)>0) \\
= & 2 \rho^{2}\left\{-\exp (1 / 2)\left[3 \rho^{3}+6 \rho^{2}+\left(24+\frac{2}{\exp (1 / 2)}\right) \rho\right]+24\right\} \\
\geq & 2 \rho^{2}\left\{-\exp (1 / 2)\left[3 \rho^{3}+6 \rho^{2}+\left(24+\frac{2}{\exp (1 / 2)}\right) \rho\right]_{\rho=1 / 2}+24\right\} \\
= & 2 \rho^{2}\left[23-\frac{111}{8} \exp (1 / 2)\right]\left(\approx 0.248 \rho^{2}\right)>0
\end{aligned}
$$

and

$$
\begin{aligned}
N_{9}(\rho, 4) & =\left\{-6 \rho\left[\rho^{2}+8 \rho+16\right] \exp \left(\frac{4 \rho}{4+\rho}\right)-2 \rho^{2}+96\right\} \rho^{2} \\
& >\left\{-6 \rho\left[\rho^{2}+8 \rho+16\right] \exp \left(\frac{4 \rho}{4+\rho}\right)-2 \rho^{2}+96\right\}_{\rho=1 / 2} \rho^{2} \\
& =\left[\frac{191}{2}-\frac{243}{4} \exp \left(\frac{4}{9}\right)\right] \rho^{2}\left(\approx 0.753 \rho^{2}\right)>0 .
\end{aligned}
$$

Hence $N_{9}(\rho, a)>0$ for $a \geq 4$ and $0<\rho \leq \bar{\rho}$. It implies that, by (4.6), $N_{6}(\rho, a)$ is a strictly increasing function of $a$ on $[4, \infty)$ for any fixed $\rho \in(0, \bar{\rho}]$. 


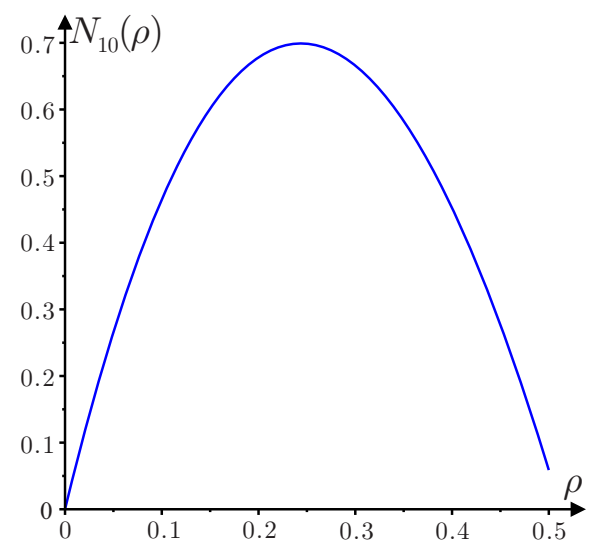

Figure 4.1: Graph of $N_{10}(\rho)$ on $(0,1 / 2)$.

Hence, by (4.4) and Lemma 3.7(iii), we conclude that $N_{7}(\rho, a)$ is a strictly increasing function of $a$ on $[4.37, \infty)$ for any fixed $\rho \in(0, \bar{\rho}]$. It follows that

$$
N_{7}(\rho, a)>N_{7}(\rho, 4.37) \equiv N_{10}(\rho)>0
$$

for $0<\rho \leq \bar{\rho}(<1 / 2)$; see Figure 4.1. So 1.13 holds for $a \geq 4.37$.

The proof of Theorem 2.5 is now complete.

\section{Acknowledgments}

The authors thank the anonymous referee for valuable suggestions which helped to improve this paper. Most of the computation in this paper has been checked using the symbolic manipulator MAPLE 16.

\section{References}

[1] K. J. Brown, M. M. A. Ibrahim and R. Shivaji, S-shaped bifurcation curves, Nonlinear Anal. 5 (1981), no. 5, 475-486.

[2] A. K. Drame and D. G. Costa, On positive solutions of one-dimensional semipositone equations with nonlinear boundary conditions, Appl. Math. Lett. 25 (2012), no. 12, 2411-2416.

[3] J. Goddard II, E. K. Lee and R. Shivaji, A double S-shaped bifurcation curve for a reaction-diffusion model with nonlinear boundary conditions, Bound. Value Probl. 2010 (2010), Art. ID 357542, 23 pp. 
[4] P. V. Gordon, E. Ko and R. Shivaji, Multiplicity and uniqueness of positive solutions for elliptic equations with nonlinear boundary conditions arising in a theory of thermal explosion, Nonlinear Anal. Real World Appl. 15 (2014), 51-57.

[5] S.-Y. Huang and S.-H. Wang, Proof of a conjecture for the one-dimensional perturbed Gelfand problem from combustion theory, Arch. Ration. Mech. Anal. 222 (2016), no. $2,769-825$.

[6] K.-C. Hung and S.-H. Wang, A theorem on S-shaped bifurcation curve for a positone problem with convex-concave nonlinearity and its applications to the perturbed Gelfand problem, J. Differential Equations 251 (2011), no. 2, 223-237.

[7] K.-C. Hung, S.-H. Wang and C.-H. Yu, Existence of a double S-shaped bifurcation curve with six positive solutions for a combustion problem, J. Math. Anal. Appl. 392 (2012), no. 1, 40-54.

[8] P. Korman and Y. Li, On the exactness of an S-shaped bifurcation curve, Proc. Amer. Math. Soc. 127 (1999), no. 4, 1011-1020.

[9] D.-C. Kuo, S.-H. Wang and Y.-H. Liang, Proofs of some results in the article: Classification and evolution of bifurcation curves for a DirichletNeumann boundary value problem and its application, Available from:

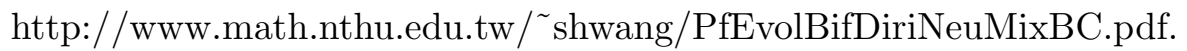

[10] T. Laetsch, The number of solutions of a nonlinear two point boundary value problem, Indiana Univ. Math. J. 20 (1970), 1-13.

[11] Y.-H. Liang and S.-H. Wang, Classification and evolution of bifurcation curves for the one-dimensional perturbed Gelfand equation with mixed boundary conditions, J. Differential Equations 260 (2016), no. 12, 8358-8387.

Da-Chang Kuo and Shin-Hwa Wang

Department of Mathematics, National Tsing Hua University, Hsinchu 300, Taiwan

E-mail address: s102021615@m102.nthu.edu.tw, shwang@math.nthu.edu.tw

Yu-Hao Liang

Department of Applied Mathematics, National Chiao Tung University, Hsinch 300, Taiwan

E-mail address: yhliang@g2.nctu.edu.tw 